\title{
DBFU-Net: Double branch fusion U-Net with hard example weighting train strategy to segment retinal vessel
}

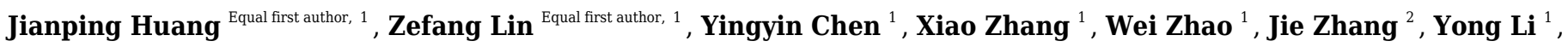 \\ Xu He ${ }^{1}$, Meixiao Zhan ${ }^{1}$, Ligong Lu ${ }^{1}$, Xiaofei Jiang ${ }^{3}$, Yongjun Peng ${ }^{\text {Corresp. } 2}$ \\ ${ }^{1}$ Zhuhai People's Hospital, Zhuhai Hospital Affiliated with Jinan University, Jinan University, Zhuhai Interventional Medical Center, Zhuhai Precision \\ Medical Center, Zhuhai, China \\ ${ }^{2}$ Zhuhai People's Hospital, Zhuhai Hospital Affiliated with Jinan University, Jinan University, Department of Nuclear Medicine, Zhuhai, China \\ ${ }^{3}$ Zhuhai People's Hospital, Zhuhai Hospital Affiliated with Jinan University, Jinan University, Department of cardiology, Zhuhai, China \\ Corresponding Author: Yongjun Peng \\ Email address: pengyongjun_zy@163.com
}

Background. Many fundus imaging modalities measure ocular changes. Automatic retinal vessel segmentation (RVS) is a significant fundus image-based method for the diagnosis of ophthalmologic diseases. However, precise vessel segmentation is a challenging task when detecting micro-changes in fundus images, e.g., tiny vessels, vessel edges, vessel lesions and optic disc edges.

Methods. In this paper, we will introduce a novel double branch fusion U-Net model that allows one of the branches to be trained by a weighting scheme that emphasizes harder examples to improve the overall segmentation performance. A new mask, we call a hard example mask, is needed for those examples that include a weighting strategy that is different from other methods. The method we propose extracts the hard example mask by morphology, meaning that the hard example mask does not need any rough segmentation model. To alleviate overfitting, we propose a random channel attention mechanism that is better than the drop-out method or the L2-regularization method in RVS.

Results. We have verified the proposed approach on the DRIVE, STARE and CHASE datasets to quantify the performance metrics. Compared to other existing approaches, using those dataset platforms, the proposed approach has competitive performance metrics. (DRIVE: F1-Score=0.8289, G-Mean=0.8995, AUC $=0.9811$; STARE: F1-Score=0.8501, G-Mean=0.9198, AUC=0.9892; CHASE: F1-Score=0.8375, GMean=0.9138, AUC=0.9879).

Discussion. The segmentation results showed that DBFU-Net with RCA achieves competitive performance in three RVS datasets. Additionally, the proposed morphological-based extraction method for hard examples can reduce the computational cost. Finally, the random channel attention mechanism proposed in this paper has proven to be more effective than other regularization methods in the RVS task. 
1 DBFU-Net: Double branch fusion U-Net with hard

2 example weighting training strategy to segment retinal

3 vessels

4

5

6

Jianping Huang ${ }^{1, \dagger}$, Zefang Lin ${ }^{1, \dagger}$, Yingyin Chen ${ }^{1}$, Xiao Zhang ${ }^{1}$, Wei Zhao ${ }^{1}$, Jie Zhang ${ }^{3}$, Yong $\mathrm{Li}^{1}, \mathrm{Xu} \mathrm{He}{ }^{1}$, Meixiao Zhan ${ }^{1}$, Ligong Lu ${ }^{1}$, Xiaofei Jiang ${ }^{2}$, Yongjun Peng ${ }^{3}$

${ }^{1}$ Zhuhai Interventional Medical Center, Zhuhai Precision Medical Center, Zhuhai People's Hospital, Zhuhai Hospital Affiliated with Jinan University, Jinan University, Zhuhai, Guangdong, P.R. China

${ }^{2}$ Department of cardiology, Zhuhai People's Hospital, Zhuhai Hospital Affiliated with Jinan University, Jinan University, Zhuhai, Guangdong, P.R. China

${ }^{3}$ Department of Nuclear Medicine, Zhuhai People's Hospital, Zhuhai Hospital Affiliated with Jinan University, Jinan University, Zhuhai, Guangdong, P.R. China

$\dagger$ These authors contributed equally to this work and should be considered co-first authors Corresponding Author:

Yongjun Peng ${ }^{3}$

79 Kangning Road, Zhuhai, Guangdong, 519000, P.R. China

Email address: pengyongjun_zy@163.com

\section{Abstract}

Background. Many fundus imaging modalities measure ocular changes. Automatic retinal vessel segmentation (RVS) is a significant fundus image-based method for the diagnosis of ophthalmologic diseases. However, precise vessel segmentation is a challenging task when detecting micro-changes in fundus images, e.g., tiny vessels, vessel edges, vessel lesions and optic disc edges.

Methods. In this paper, we will introduce a novel double branch fusion U-Net model that allows one of the branches to be trained by a weighting scheme that emphasizes harder examples to improve the overall segmentation performance. A new mask, we call a hard example mask, is needed for those examples that include a weighting strategy that is different from other methods. The method we propose extracts the hard example mask by morphology, meaning that the hard example mask does not need any rough segmentation model. To alleviate overfitting, we propose a random channel attention mechanism that is better than the drop-out method or the L2regularization method in RVS.

Results. We have verified the proposed approach on the DRIVE, STARE and CHASE datasets to quantify the performance metrics. Compared to other existing approaches, using those dataset platforms, the proposed approach has competitive performance metrics. (DRIVE: F1- 
40 Score $=0.8289$, G-Mean=0.8995, AUC=0.9811; STARE: F1-Score=0.8501, G-Mean=0.9198,

$41 \mathrm{AUC}=0.9892$; CHASE: F1-Score=0.8375, G-Mean=0.9138, AUC=0.9879).

42 Discussion. The segmentation results showed that DBFU-Net with RCA achieves competitive

43 performance in three RVS datasets. Additionally, the proposed morphological-based extraction

44 method for hard examples can reduce the computational cost. Finally, the random channel

45 attention mechanism proposed in this paper has proven to be more effective than other

46 regularization methods in the RVS task.

47

48

49

50

51

52

53

54

55

56

57

58

59

60

61

62

63

64

65

66

67

68

69

70

71

72

73

74

75

76

77

78

79

keywords: vessel segmentation, deep learning, fundus image, hard examples, random channel attention mechanism

\section{Introduction}

Diabetic retinopathy (DR) refers to progressive retinal damage that occurs in people with diabetes. This disease may cause vision loss, has no symptoms in the early stages, and usually develops rapidly [1]. The narrowing of small blood vessels in the retina is a specific indicator of the disease, thus the ophthalmologists can make a diagnosis by analyzing the retinopathy [2]. However, due to the high prevalence of diabetes and the lack of human experts, screening procedures are expensive and time-consuming for clinics. Thus, reliable automatic analysis methods of retinal images will greatly reduce the workload of ophthalmologists and contribute to a more effective screening procedure [3, 4]. Therefore, a computer-aided automated retinal vessel segmentation (RVS) is highly desirable in many cases [5]. Automated RVS is a well-regarded method in ophthalmologic image analysis. Automatic computer-aided medical image analysis has been introduced to improve the performance and efficiency of RVS in recent years, thanks to advances in image processing and artificial intelligence. We divide these methods into two categories: learning-based and non-learningbased methods. Machine learning-based methods can further be categorized as supervised and unsupervised methods. The algorithm we propose in this paper is a supervised deep learning method.

\subsection{Related work}

The retinal vessel extraction problem is comparable to the segmentation of foreground and background in fundus image The related research can be traced back to the late 1980's [6]. In recent years, machine learning methods have become more popular and successful in natural image processing. An increasing number of medical image research projects have focused on learning-based algorithms.

\subsubsection{Non-learning-based methods}

Non-learning-based segmentation methods are often limited to an accurate artificial description capability, while learning-based methods are limited to training data [7]. For example, Sheng et al. proposed a robust and effective approach that qualitatively improves the detection of lowcontrast and narrow vessels [8]. Rather than using the pixel grid, they used a super-pixel as the elementary unit of the vessel segmentation scheme. Khan et al. presented a couple of contrast- 
80

81

82

83

84

85

86

87

88

89

90

91

92

93

94

95

96

97

98

99

100

101

102

103

104

105

106

107

108

109

110

111

112

113

114

115

116

117

118

119

120

sensitive measures to boost the sensitivity of existing RVS algorithms [9]. They applied a scalenormalized detector that detects vessels regardless of size. A flood-filled reconstruction strategy was adopted to get a binary output. Sazak et al. introduced a new approach based on mathematical morphology for vessel enhancement, which combines different structuring elements to detect the innate features of vessel-like structures [10]. The non-learning-based methods can avoid complex training processes, but their segmentation performance is not as good as learning-based algorithms.

\subsubsection{Unsupervised methods}

An unsupervised learning algorithm can be executed without requiring manual annotations. Currently, most RVS tasks (based on unsupervised methods) in the literature are performed using Hessian matrices, morphological operations, or similar methods. Neto et al. used statistics of spatial dependency and probability to coarsely approximate a vessel map with an adaptive local thresholding scheme. The coarse segmentation is then refined through curvature analysis and morphological reconstruction to reduce pixel mislabeling and to better estimate the retinal vessel tree [11]. Yan et al. proposed an accelerated matrix decomposition mechanism, which could be used to boost not only the original Hessian-based multi-scale approach, but also singular value decomposition-based algorithms [12]. Yin et al. used an orientation-aware detector (OAD) to accurately extract retinal vessels. The detector has a linearly elongated structure and was designed according to the locally oriented intrinsic property of vessels [1]. Researchers used the OAD to extract the shape of a vessel with no assumptions on the parametric orientations of the vessel shape. Thus, the various orientations of vessels can be efficiently modeled by an energy distribution of a Fourier transformation. The major advantage of using unsupervised algorithms is that they do not require manual labeling for training, however their performance is not as good as most supervised algorithms.

\subsubsection{Supervised methods}

Generally, the performance of supervised segmentation methods is better than that of unsupervised methods, generally because these methods were all based on already classified data for segmentation [13]. The supervised learning-based approaches can further be classified into two groups: shallow learning-based approaches and artificial neural network-based approaches. Currently, shallow learning-based segmentation methods utilize handcrafted features for prediction. Palanivel et al. proposed a novel retinal vasculature segmentation method based on multifractal characterization of the vessels to minimize noise and enhance the vessels during segmentation [14]. The Holder exponents are computed from Gabor wavelet responses, which is an effective way to segment vessels and a novel feature of the method. However, the local regularity of the vessel structures extracted, based on Holder exponents, can easily miss small vessels features.

In fundus imaging, artificial neural networks were first used for classification tasks [15]. After the introduction of FCNs, a growing number of researchers turned to deep convolutional neural networks for segmentation tasks. Since then, several attempts have been made by introducing deep convolution neural network framework algorithms to segment retinal vessels. Yang et al. proposed a multi-scale feature fusion RVS model based on U-Net, called MSFFU-Net, that 
121

122

123

124

125

126

127

128

129

130

131

132

133

134

135

136

137

138

139

140

141

142

143

144

145

146

147

148

149

150

151

152

153

154

155

156

157

158

159

introduces an inception structure into the multi-scale feature extraction encoder part of the process. Additionally, a max-pooling index was applied during the upsampling process in the feature fusion decoder of the improved network [16]. Leopold et al. had compiled various key performance indicators (KPIs) and state-of-the-art methods that were applied to the RVS task; this framed computational efficiency-performance trade-offs under varying degrees of information loss using common datasets and introduced PixelBNN [17]. Thus, highly efficient deep learning methods for automating the segmentation of fundus morphologies was discovered. A retinal image segmentation method is also proposed by Li et al, , called the MAU-Net [18], that takes advantage of both modulated deformable convolutions and dual attention modules to realize vessel segmentation based on the U-net structure. Kromm et al. developed a novel deep learning method for vessel segmentation and centerline extraction of retinal blood vessels based on the Capsule network in combination with an Inception architecture [19]. Ribeiro et al. explored the implementation of two ensemble techniques for RVS, Stochastic Weight Averaging and Snapshot Ensembles [20]. Adarsh et al. implemented an auto encoder deep learning network model based on residual paths and a U-Net that effectively segmented retinal blood vessels [21]. Guo et al. presented a multi-scale supervised deep learning network with short connections (BTS-DSN) [22] for vessel segmentation. Researchers used short connections to transfer semantic information between side-output layers. Bottom-top short connections pass from lowlevel semantic information to high-level information to refine the results to high-level sideoutputs. The top-bottom short connection transmits structural information to the low-level to reduce the noise of low-level side-outputs. Yan et al. explored the segments of both thick and thin vessels separately by proposing a three-stage deep learning model [23]. The vessel segmentation task is divided into three stages: thick vessel segmentation, thin vessel segmentation, and vessel fusion. Zhao et al. proposed a new approach as a step of postprocessing [24] to improve the existing method by formulating the segmentation as a matting problem. A trimap is obtained via a bi-level thresholding of the score map using existing methods, which is instrumental in focusing the attention to the pixels of these unknown areas. Among these ANN methods, [16], [19], [21], [22], [23] have researched on multi-scale features, [18] has researched attention mechanisms, [20] has researched ensemble strategy methods, [17] has researched the dependency between pixels, and [24] has researched post-processing methods. These studies can improve the accuracy of segmentation models. But they did not focus on many difficult samples in the training process, so the overall segmentation performance (F1 Score) of these methods is not good enough.

Current supervised algorithms have produced some excellent results in RVS. The segmentation performance on optic disc, thin vascular, and lesion areas, however, remain unsatisfactory. The output probability map of models in optic disc, thin vascular, and lesion areas are close to 0.5 , and thus we call these examples hard examples. The mask of hard examples can guide the training process of the models. Current methods to extract hard example masks, however, need rough segmentation results to set a probability range for increasing the computational complexity 
160 of the algorithm. In addition, the data volume of RVS datasets, which are scarce, will lead to

161

162

163

164

165

166

167

168

169

170

171

172

173

174

175

176

177

178

179

180

181

182

183

184

185

186

187

188

189

190

191

192

193

194

195

196

problems of model overfitting, limiting the use of deep learning algorithms.

\subsection{Contributions}

To overcome problems described at 1.1.3, we directly extract the hard example mask from the ground truth via morphology. Then, we matched the hard example masks to design the double branch fusion U-Net (DBFU-Net), where one branch was trained by cross entropy and the other branch was trained by the improved cross entropy that applies weights to the hard example. In addition, we propose a random channel attention mechanism to prevent overfitting. The main contributions of this paper are listed as follows:

-To overcome overfitting, we propose a novel regularization method, called Random Channel Attention Mechanism (RCA), that applies random weights to hidden layers channelwise. The performance of the proposed regularization method is better than dropout and L2 regularization.

-To extract the hard example of RVS, we propose a hard example extraction method based on image morphology.

- We propose a DBFU-Net that fuses with two decoder branches, such that one of the branches pays more attention to the hard example to improve the segmentation performance.

As an overview, the details of the proposed method are introduced in Section 2. Section 3 describes the experimental process and discusses the segmentation results. The conclusion of the paper is provided in section 4 .

\section{Methods}

RVS is challenging when applying deep learning of models of the optic disc, thin vascular, and lesion region; this is largely because the pixels of these areas are not distinct from each other. Furthermore, the model is subject to overfitting during the training process due to data scarcity. To improve segmentation performance in a hard example and alleviate overfitting, we propose the DBFU-Net trained by RCA. The DBFU-Net training process requires a hard example mask. We propose a hard example mask extraction method based on morphology to reduce computational cost. In this section, we define the RVS hard example first. Then, we describe the hard example mask extraction method based on morphology and the weighting loss of hard examples. After that, we introduce the RCA regularization approach and the structural details of DBFU-Net. Finally, we describe the implementation of our method.

\subsection{Hard example extraction base on morphology}

Generally, the loss of segmentation result is computed via cross entropy in the end-to-end model train process. Each pixel is treated with equal importance, however, the hard segmentation region cannot be more important. To ensure the model is more focused on the hard segmentation area, we could extract hard example masks and weight the loss function. 
197 The output of the RVS model is usually a probability distribution map when the output value of 198 the pixel probability is close to 1 ; in this case, the model considers the pixel to be a blood vessel. 199 If the pixel probability value of the output is close to 0 , the model considers the pixel to be part 200 of the background. However, if the pixel probability value of the output is close to 0.5 , it is 201 difficult for the model to determine whether the pixel is a blood vessel or part of the background. 202 Hence, we call all pixels that are difficult to distinguish via modeling hard examples, described 203 by a probability values range.

204 Based on the definition of hard examples of RVS, Zhao et al. extracted the hard example mask 205 by setting a threshold range [24], shown in Fig. 1. However, hard example extractions need a 206 probability map generated by the model. Hard example masks can guide the model to focus more 207 on hard example areas in the training process; this is the reason why we would like to extract the 208 hard example mask. Thus, a model is needed to extract these hard example masks extracted by a probability range to generate a rough segmentation result, leading to an increase in calculations and to a higher computational cost. To reduce the computational complexity of the hard example mask extraction, we propose a novel method that extracts the hard example mask of the RVS

212 based on morphology, see Fig. 2. The details of the hard example mask are shown in Fig. 3.

213 Fig. 2 and Fig. 3 demonstrates that, compared to the result of using the threshold-based method 214 and the morphology operation-based method, the hard example mask extracted by both methods 215 can obtain similar results; however the proposed method is simpler and more efficient than a 216 probability threshold-based method. This is because a morphology operation-based method 217 doesn't need a probability map from a rough segmentation model. The hard example mask can 218 be described by Eq. 1.

To train the model with more of a focus on the hard example, we set hard example loss weights, which means that we add a weighting value to the overall weighting of the cross-entropy loss 222 function.

$$
\begin{gathered}
W_{h}=\text { Mask } \times \text { weight }+1, \\
\text { loss }=\sum[y \log \hat{y}+(1-y) \log (1-\hat{y})] \times W_{h^{\prime}}
\end{gathered}
$$

where $W_{h}$ is a hard example weight and weight is a hyperparameter. According to the Eq. 3, compared with a cross entropy loss function, the hard example loss will increase because of the 


\subsection{Random channel attention mechanism.}

230 Overfitting is a common problem when training deep neural networks because of the lack of 231 training data or the relatively simple network. To solve this problem, in addition to data 232 augmentation, regularization is an effective method to alleviate overfitting, e.g., dropout [25] or

233

234

235

236

237

238

239

240

241

242

243

244

245

246

247

248

249

250

251

252

253

254

255

256

257

258

259

260

261

262

263

264

265

266

267

L2 regularization. In this paper, we propose a novel regularization algorithm where the feature channels are randomly weighted during the model training phase. Different from channel attention mechanisms that provide specific weights on the feature channel, the random channel attention (RCA) mechanism allocates different and random weights to each channel. The method is demonstrated in Fig. 4. Therefore, the robustness of the deep learning model is enhanced due to the nature of the randomness of the training process when compared to the dropout method, which involves randomly setting the output of each hidden neuron to zero with a certain probability. RCA is a soft method that involves only weighting the feature channel and ensures that a deep learning model is easier to train. The experiment in part 3 demonstrates that the speed of training by RCA converges faster than dropout and L2 regularization. Furthermore, the performance of the model trained by RCA is better than that of the model trained by other regularization methods.

\subsection{Double branch fusion U-Net}

Experiments show that paying more attention to hard examples during the training process can improve segmentation results of a hard example region, however, this process will bring more false positive samples in the holistic segmentation result. To improve the performance of a hard example region segmentation, without increasing the false positive rate, we assume that the model is composed of a single encoder, two decoders, and a single fusion layer. The encoder is used to extract features from the original image, and the segmentation probability map generated by the decoder is based on features extracted by the encoder. One of the decoders is trained by cross entropy and weighted by hard example weights, while the other decoder is only trained by cross entropy. To fuse two decoder features, a fusion layer combines two branch decoders to generate the final segmentation result.

Inspired by U-net [26], we propose the DBFU-Net with an overview architectural diagram as shown in Fig. 5. The network is composed of three parts that perform specific tasks: an encoder sub-network extracts high-level image features, two-decoder sub-networks generate a rough segmentation result, and one fusion layer combines features extracted by the two decoders to compute the final segmentation result. Each branch has a loss function to optimize all parameters. Like the deeply supervised training strategy, the proposed method will avoid the risk of increasing the network's depth increasing the complexity of the optimization. The model's focus needs to be on hard examples, therefore, one of the decoder branches is trained by the loss function (Eq. 3). The block used for the proposed DBFU-Net is improved by the res-block inspired by Link-Net [27], which combines RCA to alleviate overfitting. The structure of the Res-block of the DBFU-Net is shown in Fig. 6.

\subsection{Implementation details}

Peer] Comput. Sci. reviewing PDF | (CS-2021:01:57330:5:1:NEW 5 Jan 2022) 
268 We provide implementation details, which mainly includes preprocessing, training the first 269 decoder, training the second decoder, training the fusion layer, and post processing. The detailed 270 description of each step is listed as follows:

271 Preprocessing. To fit input data into the RVS model, we apply a preprocessing step to the 272 fundus image. Because the blood vessels manifest high contrast in the green channel [1], we 273 extract the green channel images, given an RGB fundus image. Since the network has a 274 downsampling factor of 5 , the size of the input image should be divisible by $2^{5}$, therefore we had 275 to pad the input image with multiples of $2^{5}$. To adjust image contrast, we use contrast limited 276 adaptive histogram equalization to enhance the input image. Then, we utilize a morphology 277 method to obtain the hard example mask according to the label.

278 The lack of labeled data is one of the most difficult problems for RVS. Consider the DRIVE 279 dataset as an example, the training set of the DRIVE dataset only contains 20 pictures. For the 280 supervised algorithms, the use of data augmentation technology alleviates the problem of data 281 scarcity. In this paper, we augment the training data using rotating, mirroring, and translating 282 operations. Additionally, we use random elastic deformations to augment the training data to 283 obtain more morphological characteristics of vessel. The process of random elastic deformation 284 is shown in Fig. 7.

285 Training the first decoder. We train the first decoder to obtain the parameter for the encoder. In 286 this process, the learning rate is initially set to $7 \mathrm{e}-4$ and multiplied by $1 / 3$ every $1 / 3$ epoch; the 287 batch size is 2 . The network model is trained for 12 epochs with an SGD optimizer, and the 288 parameters are randomly initialized by he-normal [28].

289 Training the second decoder. To ensure the model focuses more on hard examples, we train the 290 second decoder with cross entropy after weighting the hard example. In this process, we freeze 291 the parameters of the encoder, and the parameters of the second decoder are randomly initialized 292 by he-normal. The learning rate is initially set to $7 \mathrm{e}-4$ and multiplied by $1 / 3$ every $1 / 3$ epoch, and 293 the batch size is 2 . The network model is trained for 12 epochs with an SGD optimizer. After 294 that, we have fine-tuned all the parameters for 8 epochs with a learning rate of $5 \mathrm{e}-5$.

295 Training the fusion layer. The first decoder can obtain fundus vessels from the features that are 296 extracted by the encoder, but the second decoder focuses more on thin vessels. Therefore, we 297 train the fusion layer to combine features from the two branches to obtain a segmentation result 298 that is better than using only one branch. In this process, we freeze all parameters except fusion 299 layers made of parameters randomly initialized by he-normal. The learning rate is initially set to $3007 \mathrm{e}-4$ and multiplied by $1 / 3$ every $1 / 3$ epoch; the batch size is 2 . The network model is trained for 3016 epochs with the SGD optimizer. After that, we fine-tune all parameters for 4 epochs with a 302 learning rate of $5 \mathrm{e}-5$.

303 Post processing. The range of segmentation probability map generated by model is $[0,1]$. To 304 305 306 ensure the segmentation result is in the form of gray images, we normalize the segmentation probability map to a range of $[0,255]$. The final probability map is converted into binary images by applying the global threshold segmentation algorithm. Different segmentation performances will be achieved when applying different binarization thresholds. We choose the threshold that 
308 has the highest F1-score for the validation set as the optimal threshold value. The best threshold 309 of the model varies due to different output results, which can reflect the best performance of the 310 various models.

\section{Results}

313

In this section we will present our experimental datasets and settings, as well as the RVS

314 performance indicator and experiment results.

\section{3.1 Materials and experimental settings}

316 Similar to most RVS work, we evaluated the proposed method using DRIVE (Digital Retinal

317 Images for Vessel Extraction) [2], STARE (Structured Analysis of the Retina) [29] and CHASE

318 (Child Heart Health Study in England) [30] datasets, which are shown in Fig. 8. We find that

319 different datasets have different data distribution characteristics. The DRIVE dataset contains 40

320

321

322

323

324

325

326

327

328

329

330

331

332

333

334

335

336

337

338

339

340

341

342

343 color images with a resolution of $565 \times 584$, which are captured at $45^{\circ}$ field of view (FOV) and divided into a training set and a test set equally. The STARE dataset has 20 color fundus images that are captured at $35^{\circ} \mathrm{FOV}$. The resolution of each image is $700 \times 605$. The CHASE dataset provides 14 paired color images with a resolution of $999 \times 960$. The images were collected from both the left and right eyes of school children. Note that in these datasets, each image has two manually labeled binary images with an FOV mask. We choose the binary images of the first observer as the ground truth. In the experiment using the DRIVE dataset, we tested model by the official test set. We perform the five-fold and four-fold cross-validation for the STARE and CHASE datasets because they have no official test datasets. In all experiments, we divided $10 \%$ of the training set as the validation set and select the model with the best performance in the validation set for testing to determine the threshold of binarization based on the selected model. The experimental computer has a Windows Server 2016 operating system running on two Intel Core Xeon Gold 6234 CPUs and two NVIDIA Tesla V100 Graphics Processing Units (GPUs).

\subsection{Performance measurements}

RVS is a pixel-level binary classification problem in which each pixel can be divided as vessel

and non-vessel pixels. The positive examples are the vessel pixels and the negative examples are non-vessel pixels. We can evaluate the RVS performance based on a confusion matrix that includes a TP (Ture Positive), TN (Ture Negative), FP (False Positive) and FN (False Negative). Then, based on the evaluation, we can generate the receiver operating characteristic (ROC) curve [31] to calculate the area under the ROC curve (AUC). In this paper, the RVS performance is measured by F1-score, sensitivity (Se), specificity (Sp), accuracy (Acc), G-mean, Matthews Correlation Coefficient (MCC) and AUC, which are defined as follows:

$$
\begin{gathered}
F 1 \text { Score }=\frac{2 \times T P}{2 \times T P+F P+F N} \\
S n=\frac{T P}{T P+F N}
\end{gathered}
$$


344

345

346

347

348

349

350

351

352

353

354

355

356

357

358

359

360

361

362

363

364

365

366

367

368

369

370

371

372

373

374

375

376

377

378

379

380

381

382

$$
\begin{gathered}
S p=\frac{T N}{T N+F P} \\
A c c=\frac{T P+T N}{T P+T N+F P+F N} \\
G-M e a n=\sqrt{S n \times S p} \\
M C C=\frac{T P \times T N}{\sqrt{(T P+T N)(T P+F N)(T N+F P)(T N+F N)}}
\end{gathered}
$$

The ratio of positive and negative examples is 1 to 9 according to the statistics of the data set. Therefore, the Acc will reach $90 \%$ but the $\mathrm{Sn}$ is 0 when all pixels are classified as negative examples. That is the reason why making ACC as the main evaluation indicator [32] is inappropriate. We should consider both Sn and Sp when measuring RVS performance because Sn and Sp only focuses on positive and negative examples. The MCC and F1-Score consider all categories of possible classification situations at the same time. Therefore, both the MCC and F1-Score can be used in the case of uneven samples; this model is commonly regarded as a balanced evaluation indicator. In this paper, all RVS indicators were calculated using only pixels inside FOVs over all the test images.

\subsection{Experimental results}

In this part, we conducted ablation experiments of DBFU-Net and show the performance of DBFU-Net on DRIVE, STARE and CHASE datasets.

\subsubsection{Comparison with other regularization method}

To compare the performance of different regularization methods, we used U-Net with res-block (single branch model) and DBFU-Net to compare the performance of model training, by different regularization methods and by using training models with no regularization method in three datasets. The comparison results on DRIVE, STARE and CHASE datasets are shown in table 1 . The dropout rate is set to 0.5 , the $\mathrm{L} 2$ regularization parameter is set to $1 \mathrm{e}-3$, the mean of weight is set to 1 and var is set to 0.5 of the RCA in all experiments. To show that the RCA can have better regularization capabilities on different models, we also used HR-Net [33] for comparative experiments.

\subsubsection{Comparison of hard example weighting strategy.}

To verify the effectiveness of the hard example weighting strategy, we conducted a comparative experiment using DRIVE, STARE and CHASE datasets, to compare the performance of a single-branch model. The two-branch model does not use the hard example weighting training strategy (DBFU-Net-NH), decoder 1 of DBFU-Net, decoder 2 of DBFU-Net or the DBFU-Net. The experimental results are shown in table 2. The second branch of DBFU-Net-NH was trained by cross entropy. All controlled experiments in this section use the RCA regularization method.

\subsubsection{Comparison with dice loss, focal loss}

The second decoder of DBFU-Net can focus on the hard example in the training process. Focal loss [34] can also pay more attention to hard example pixels, shown in Eq. 10. Dice loss [35] is proposed for uneven data distributions; the effect of focal loss and dice loss in the training process are like the proposed training strategy, which weights hard examples as shown in Eq. 12. Therefore, we compared the proposed hard example weighting strategy with focal loss and dice loss. The parameter $\gamma$ of focal loss was set to 2 and the parameter $\varepsilon$ of dice loss was set to 1 e- 5 . 
383 The second decoder was trained by the hard example weighting strategy, focal loss, and dice

384

385

386

387

388

389

390

391

392

393

394

395

396

397

398

399

400

401

402

403

404

405

406

407

408

409

410

411

412

413

414

415

416

417

418

419

loss. Then we used the result of the fusion layer as the final comparative result. In addition, we compared the performance of the second decoder of DBFU-Net and the single branch model that was trained by focal loss and dice loss. The comparative experiment results for the DRIVE, STARE and CHASE datasets are shown in table 3. SB-F represents a single branch model trained by focal loss; SB-D represents a single branch model trained by dice loss. DBFU-Net-F represents a DBFU-Net trained by focal loss. DBFU-Net-D represents a DBFU-Net trained by dice loss; decoder 2 represents a second decoder of DBFU-Net. All controlled experiments in this section use an RCA regularization method.

$$
\begin{gathered}
F L\left(p_{t}\right)=-\left(1-p_{t}\right)^{\gamma} \log \left(p_{t}\right) \\
p_{t}=\left\{\begin{array}{r}
p, \quad \text { if } y=1 \\
1-p, \quad \text { otherwise },
\end{array}\right.
\end{gathered}
$$

where $\gamma$ is a hyperparameter, $p$ is output possibility of deep learning model, $y$ is label.

$$
\begin{gathered}
\text { Dice loss }=1-\frac{2 I+\varepsilon}{U+\varepsilon} \\
I=\sum t_{i} y_{i} \\
U=\sum t_{i}+\sum y_{i},
\end{gathered}
$$

where $t_{i}$ is label, $y_{i}$ is output possibility of deep learning model, $\varepsilon$ is a hyperparameter.

\section{Discussion}

This section analyzes the results of the ablation experiment of DBFU-Net. We analyzed the effect of RCA and hard example weighting training strategy on the ablation experiment. We also compared the performance of DBFU-Net to other published methods.

\subsection{Comparison with other regularization method}

It is easy to overfit when training the RVS deep learning model, that is why we needed to come up with an effective regularization method. The proposed RCA is an effective regularization method, shown in Fig. 9, which compares it to the Drop out and L2 regularization training curves.

Each regularization method shown in table 1, Fig. 9 and Fig. 10, performs similarly on the different datasets. Compared with a single branch model, the validation loss of DBFU-Net converges more slowly because DBFU-Net has more parameters. In the blank contrast group of a single branch model and DBFU-Net method without any regularization method, training loss can always converge given an increase in the count of the iteration; validation loss nevertheless quickly rises after a certain degree of convergence, that is an obvious overfitting phenomenon. Training loss and validation loss can still maintain a stable convergence state when iterating for a long period of time in the dropout experimental ground. However, the stable convergence value of validation loss is at a relatively high level, so the segmentation effect is not well. In the L2 regularization experimental group, the validation loss can converge steadily and concurrent with the training loss at a stable convergence state. But the validation loss will rise and the training 
420 loss will converge after more experiment iterations, that is the phenomenon of overfitting.

421 Proposing the RCA regularization method can ensure that training loss and validation loss

422 converge rapidly and at the same time. The validation loss can maintain a steady state with

423 increased train steps. The best validation loss of a model trained by RCA is to lower the models

424 trained by other regularization methods. From table 1, the segmentation performance of the

425 model trained by RCA is better than that for the other methods in the three datasets. In addition,

426 we found that the HR-Net trained by RCA can obtain better performance than other

427 regularization methods. Therefore, we can draw the conclusion that the proposed RCA

428 regularization method is better than other regularization methods.

429

430

431

432

433

434

435

436

437

438

439

440

441

442

443

444

445

446

447

448

449

450

451

452

453

454

455

456

457

458

459

\subsection{The effective of hard example weighting training strategy.}

According to table 2, the segmentation performance of DBFU-Net ranks first. The best threshold of the second decoder is the one that is higher than the contrasting result. Because the second decoder was trained using a hard example weighting strategy, the decoder paid more attention to the areas that could be a vessel, which improved the segmentation recall result, but adds more false positive points. To obtain a better segmentation result for the comprehensive performance index F1-Score, a higher threshold is required to filter the false positive points. Although the performance of the DBFU-Net-NH is worse than that of DBFU-Net, it is better than that of a model with a single branch; this is generally because DBFU-Net-NH contains more parameters. Therefore, we can draw the conclusion that a hard example weighting training strategy can improve segmentation performance. The output probability distribution maps of the first decoder, second decoder, and final fusion layer is shown in Fig. 11. The contrast of the final fusion output probability map and output of the double decoders is shown Fig. 12.

According to Fig. 11 and Fig. 12, DBFU-Net detects more positive examples than the model with a single decoder. It reduces the false positive rate when compared with the second decoder. In other words, DBFU-Net can combine the advantages of the first decoder and the second decoder, reducing the impact of their respective shortcomings.

Focal loss also pays more attention to hard example pixels; Dice loss is proposed for the uneven data distribution. The effect of focal loss and dice loss in the training process is like the proposed training strategy. Thus, we can compare the hard example weighting training strategy with focal loss and dice loss. According to table 3, we found that the performance of the DBFU-Net trained by a hard example weighting training strategy is better than other methods, especially in terms of recall. Therefore, we can draw a conclusion that the ability of attention on the hard example of the proposed method is better than that of focal loss and dice loss. In addition, the recall and the best of threshold of a single branch model was trained by focal loss and the second decoder of the DBFU-Net are significantly higher than other control results because the focal loss and hard example weighting training strategy can pay attention to hard example area, but the recall of the second decoder of DBFU-Net is higher than that of the single branch model trained by focal loss. Therefore, we can draw the conclusion that using hard example weighting training strategy can pay attention to hard example area more efficiently than focal loss in RVS. 
460

461

462

463

464

465

466

467

468

469

470

471

472

473

474

475

476

477

478

479

480

481

482

483

484

485

486

487

488

489

490

491

492

493

494

495

496

497

498

\subsection{Comparison against existing methods}

As shown in table 4, table 5 and table 6, we compared the proposed method with those of stateof-the-art methods using the DRIVE, STARE and CHASE datasets, "-" in table means that the values are not given in these papers. DBFU-Net performs the best among those methods in terms of the F1-score, Sn, G-mean and MCC, which indicates that when compared to other approaches, the DBFU-Net shows state-of-the-art efficiency. The ACC is the third highest for the DRIVE dataset. Even though our approach performs marginally worse than other methods in terms of Sp, it significantly outperforms these methods from the view of other metrics, especially for the F1score, which is considered as the primary metric in RVS. Additionally, Acc and Sp are considered reference indicators due to the nature of one-sidedness. Therefore, we can conclude that our proposed method is superior to other methods. According to table 4, table 5 and table 6 , the proposed DBFU-Net achieves state-of-the-art performance for the three datasets tested. Fig. 13 shows the performance of the method on hard examples. The image shows that in the optic disc area, our method avoids the situation where the edge of the optic disc is predicted to be a blood vessel. Our method shows a better segmentation performance than other methods in the small vascular area. Additionally, our segmentation results were not affected by retinal spots and have obtained lower FP in the lesion area when compared to the segmentation performances of other methods.

\subsection{Cross-training experiment}

The cross-training experiments reflects the robust performance of the proposed model in realistic situations [40]. Models with good robust performance can be applied to many realistic situations. The statistical results of the cross-training experiment using the three datasets are shown in table 7, "-" in table means that the values are not given in these papers. Compared with other methods, the proposed method had obtained the highest ACC, Se, F1 Score, G-Mean, MCC and AUC. The cross-training experiment not only showed that the proposed method can be applied to real-world situations and reflect that the robustness of DBFU-Net is better than that of a single branch model. In addition, in the experimental group of DBFU-Net and a single branch, the robust performance of the model trained via RCA regularization is better than the model trained by L2 regularization or drop out. Because the model was randomly introduced when using RCA training, the robustness of the model was able to be increased.

\section{Conclusions and feature work}

This paper aims at proposing a novel deep learning architecture, DBFU-Net, to segment retinal vessels. To avoid overfitting, we propose to apply RCA and to randomly weight each feature map channel. Hard example masks were introduced to guide the model to pay more attention to the edge of large vessels and thin vessel areas. To reduce the computational cost of extracting the hard example mask, we propose a novel hard example extraction method based on morphology. The experiment proved that the second training decoder achieves a performance gain when 
499

500

501

502

503

504

505

506

507

508

509

510

511

512

513

514

515

516

517

518

519

520

521

522

523

524

525

526

527

528

529

530

531

532

533

534

535

536

537

538

weighting hard examples due to the loss function. Our proposed method also obtained state-ofthe-art results for DRIVE, STARE and CHASE dataset.

We plan to examine two additional aspects in the future. First, hard example weighting is proven to be effective for RVS. We will use this method and combine it with other segmentation models for other segmentation tasks as well. DBFU-Net is a double branch model that is composed of 4 parts. Moreover, the computational cost of the hard example weighting strategy is greater than that of focal loss and dice loss because morphological operations bring additional computational costs. Hence, we will explore new methods that are less computationally expensive or based on hard example weighting training strategy.

\section{Acknowledgements}

This study is supported by the National Key Research and Development Program of China (Grant No. 2017YFA0205200), the National Natural Science Foundation of China (Grant No. 81901857), Natural Science Foundation of Guangdong Province, China (No. 2018A030313074).

\section{References}

[1] B. Yin, H. Li, B. Sheng, X. Hou, Y. Chen, W. Wu, P. Li, R. Shen, Y. Bao, W. Jia, Vessel extraction from non-fluorescein fundus images using orientation-aware detector, Medical Image Analysis, 26 (2015) 232-242.

[2] J. Staal, M.D. Abramoff, M. Niemeijer, M.A. Viergever, B. Van Ginneken, Ridge-based vessel segmentation in color images of the retina, IEEE Transactions on Medical Imaging, 23 (2004) 501-509.

[3] N.S. G. Azzopardi, M. Vento, N. Petkov, Trainable cosfire filters for vessel delineation with application to retinal images, Medical image analysis, 19 (2015).

[4] Y.Y. S. Wang, G. Cao, B. Wei, Y. Zheng, G. Yang, Hierarchical retinal blood vessel segmentation based on feature and ensemble learning, Neurocomputing, 149 (2015) 708-717. [5] R.P. E. Ricci, Retinal blood vessel segmentation using line operators and support vector classification, IEEE transactions on medical imaging, 26 (2007) 1357-1365.

[6] S. Chaudhuri, S. Chatterjee, N. Katz, M. Nelson, M. Goldbaum, Detection of blood vessels in retinal images using two-dimensional matched filters, IEEE Transactions on Medical Imaging, 8 (1989) 263-269.

[7] F. Li, C. Wang, X. Liu, Y. Peng, S. Jin, A Composite Model of Wound Segmentation Based on Traditional Methods and Deep Neural Networks, Computational Intelligence and Neuroscience, 2018 (2018) 4149103. [8] B. Sheng, P. Li, S. Mo, H. Li, X. Hou, Q. Wu, J. Qin, R. Fang, D.D. Feng, Retinal Vessel Segmentation Using Minimum Spanning Superpixel Tree Detector, IEEE Transactions on Cybernetics, 49 (2019) 2707-2719.

[9] M.A.U. Khan, T.M. Khan, T.A. Soomro, N. Mir, J. Gao, Boosting sensitivity of a retinal vessel segmentation algorithm, Pattern Analysis and Applications, 22 (2019) 583-599. 
539 [10] Ç. Sazak, C.J. Nelson, B. Obara, The multiscale bowler-hat transform for blood vessel

540 enhancement in retinal images, Pattern Recognition, 88 (2019) 739-750.

541 [11] L. Câmara Neto, G.L.B. Ramalho, J.F.S. Rocha Neto, R.M.S. Veras, F.N.S. Medeiros, An

542 unsupervised coarse-to-fine algorithm for blood vessel segmentation in fundus images, Expert

543 Systems with Applications, 78 (2017) 182-192.

544 [12] Y. Zhang, J. Lian, L. Rong, W. Jia, C. Li, Y. Zheng, Even faster retinal vessel segmentation

545 via accelerated singular value decomposition, Neural Computing and Applications, 32 (2020)

546 1893-1902.

547 [13] S. Akbar, M. Sharif, M.U. Akram, T. Saba, T. Mahmood, M. Kolivand, Automated

548 techniques for blood vessels segmentation through fundus retinal images: A review, Microscopy

549 Research and Technique, 82 (2019) 153-170.

550 [14] D.A. Palanivel, S. Natarajan, S. Gopalakrishnan, Retinal vessel segmentation using

551 multifractal characterization, Applied Soft Computing, 94 (2020) 106439.

552 [15] K. Akita, H. Kuga, Pattern Recognition Of Blood Vessel Networks In Ocular Fundus

553 Images (SPIE, 1982).

554 [16] D.L. Yang, G.; Ren, M.; Xu, B.; Wang, J, A Multi-Scale Feature Fusion Method Based on 555 U-Net for Retinal Vessel Segmentation, Entropy, 22 (2020).

556 [17] H.A. Leopold, J. Orchard, J.S. Zelek, V. Lakshminarayanan, PixelBNN: Augmenting the

557 PixelCNN with batch normalization and the presentation of a fast architecture for retinal vessel

558 segmentation, 2017), pp. arXiv:1712.06742.

559 [18] H. Li, Y. Wang, C. Wan, J. Shen, Z. Chen, H. Ye, Q. Yu, MAU-Net: A Retinal Vessels

560 Segmentation Method, 2020 42nd Annual International Conference of the IEEE Engineering in

561 Medicine \& Biology Society (EMBC)2020), pp. 1958-1961.

562 [19] C. Kromm, K. Rohr, Inception Capsule Network for Retinal Blood Vessel Segmentation

563 and Centerline Extraction, 2020 IEEE 17th International Symposium on Biomedical Imaging

564 (ISBI)2020), pp. 1223-1226.

565 [20] A. Ribeiro, A.P. Lopes, C.A. Silva, Ensemble Learning Approaches for Retinal Vessel

566 Segmentation, 2019 IEEE 6th Portuguese Meeting on Bioengineering (ENBENG)2019), pp. 1-

5674.

568 [21] R. Adarsh, G. Amarnageswarao, R. Pandeeswari, S. Deivalakshmi, Dense Residual

569 Convolutional Auto Encoder For Retinal Blood Vessels Segmentation, 2020 6th International

570 Conference on Advanced Computing and Communication Systems (ICACCS)2020), pp. 280-

571284.

572 [22] S. Guo, K. Wang, H. Kang, Y. Zhang, Y. Gao, T. Li, BTS-DSN: Deeply supervised neural

573 network with short connections for retinal vessel segmentation, International Journal of Medical

574 Informatics, 126 (2019) 105-113.

575 [23] Z. Yan, X. Yang, K. Ch0eng, A Three-Stage Deep Learning Model for Accurate Retinal

576 Vessel Segmentation, IEEE Journal of Biomedical and Health Informatics, 23 (2019) 1427-1436.

577 [24] H. Zhao, H. Li, L. Cheng, Improving retinal vessel segmentation with joint local loss by

578 matting, Pattern Recognition, 98 (2020) 107068. 
579 [25] A. Krizhevsky, I. Sutskever, G.E. Hinton, ImageNet classification with deep convolutional 580 neural networks, Commun. ACM, 60 (2017) 84-90.

581 [26] F.P. Ronneberger O., Brox T, U-Net: Convolutional Networks for Biomedical Image

582 Segmentation, Medical Image Computing and Computer-Assisted Intervention - MICCAI 2015, 583 (2015).

584 [27] A. Chaurasia, E. Culurciello, LinkNet: Exploiting encoder representations for efficient 585 semantic segmentation, visual communications and image processing2017), pp. 1-4.

586 [28] K. He, X. Zhang, S. Ren, J. Sun, Delving Deep into Rectifiers: Surpassing Human-Level 587 Performance on ImageNet Classification, international conference on computer vision2015), 588 pp. 1026-1034.

589 [29] M.M. Fraz, P. Remagnino, A. Hoppe, B. Uyyanonvara, A.R. Rudnicka, C.G. Owen, S. 590 Barman, An Ensemble Classification-Based Approach Applied to Retinal Blood Vessel 591 Segmentation, IEEE Transactions on Biomedical Engineering, 59 (2012) 2538-2548.

592 [30] A.D. Hoover, V.L. Kouznetsova, M.H. Goldbaum, Locating blood vessels in retinal images 593 by piecewise threshold probing of a matched filter response, IEEE Transactions on Medical 594 Imaging, 19 (2000) 203-210.

595 [31] T. Fawcett, An introduction to ROC analysis, Pattern Recognition Letters, 27 (2006) 861596874.

597 [32] A. Khanal, R. Estrada, Dynamic Deep Networks for Retinal Vessel Segmentation, arXiv: 598 Computer Vision and Pattern Recognition, (2019).

599 [33] K.a.X. Sun, Bin and Liu, Dong and Wang, Jingdong, Deep High-Resolution Representation 600 Learning for Human Pose Estimation, Proceedings of the IEEE/CVF Conference on Computer 601 Vision and Pattern Recognition (CVPR), (2019).

602 [34] T. Lin, P. Goyal, R. Girshick, K. He, P. Dollár, Focal Loss for Dense Object Detection, 6032017 IEEE International Conference on Computer Vision (ICCV)2017), pp. 2999-3007.

604 [35] F. Milletari, N. Navab, S. Ahmadi, V-Net: Fully Convolutional Neural Networks for 605 Volumetric Medical Image Segmentation, 2016 Fourth International Conference on 3D Vision 606 (3DV)2016), pp. 565-571.

607 [36] T. Li, M. Comer, J. Zerubia, An Unsupervised Retinal Vessel Extraction and Segmentation 608 Method Based On a Tube Marked Point Process Model, ICASSP 2020 - 2020 IEEE

609 International Conference on Acoustics, Speech and Signal Processing (ICASSP)2020), pp. 13946101398.

611 [37] H. Fu, Y. Xu, S. Lin, D. Wong, J. Liu, DeepVessel: Retinal Vessel Segmentation via Deep 612 Learning and Conditional Random Field, 2016).

613 [38] J.I. Orlando, E. Prokofyeva, M.B. Blaschko, A Discriminatively Trained Fully Connected

614 Conditional Random Field Model for Blood Vessel Segmentation in Fundus Images, IEEE

615 Transactions on Biomedical Engineering, 64 (2017) 16-27.

616 [39] M. Niemeijer, J. Staal, B. Ginneken, M. Loog, M. Abramoff, Comparative study of retinal 617 vessel segmentation methods on a new publicly available database, Proc SPIE, 5370 (2004). 
618 [40] L. Zhou, Q. Yu, X. Xu, Y. Gu, J. Yang, Improving dense conditional random field for 619 retinal vessel segmentation by discriminative feature learning and thin-vessel enhancement, 620 Computer Methods and Programs in Biomedicine, 148 (2017) 13-25.

621 


\section{Table $\mathbf{1}$ (on next page)}

Table 1: Performance of models tested on DRIVE, STARE and CHASE dataset. The top results for each dataset are marked in bold. 


\begin{tabular}{|c|c|c|c|c|c|c|c|c|c|c|}
\hline \multirow{3}{*}{ Dataset } & \multirow{3}{*}{ Model } & \multirow{3}{*}{$\begin{array}{c}\text { Regularization } \\
\text { Method }\end{array}$} & \multirow{3}{*}{ Threshold } & \multirow{3}{*}{$\mathrm{ACC}$} & \multirow{3}{*}{$\mathrm{Se}$} & \multirow{3}{*}{$\mathrm{Sp}$} & \multirow{3}{*}{ F1 } & \multirow{3}{*}{ G } & \multirow{3}{*}{$\mathrm{MCC}$} & \multirow{3}{*}{ AUC } \\
\hline & & & & & & & & & & \\
\hline & & & & & & & & & & \\
\hline \multirow{14}{*}{ DRIVE } & & No & 121 & 0.9521 & 0.8116 & 0.9704 & 0.8144 & 0.8874 & 0.7998 & 0.9789 \\
\hline & Single & Dropout & 117 & 0.9531 & 0.8185 & 0.9721 & 0.8202 & 0.8920 & 0.8001 & 0.9791 \\
\hline & branch & $\mathrm{L} 2$ & 127 & 0.9550 & 0.8231 & 0.9745 & 0.8246 & 0.8956 & 0.8008 & 0.9799 \\
\hline & & $\mathrm{RCA}$ & 125 & 0.9559 & 0.8265 & 0.9762 & 0.8259 & 0.8982 & 0.8034 & 0.9805 \\
\hline & \multirow{5}{*}{ DBFU-Net } & No & 142 & 0.9412 & 0.8107 & 0.9711 & 0.8099 & 0.8873 & 0.7996 & 0.9786 \\
\hline & & Dropout & 139 & 0.9549 & 0.8199 & 0.9734 & 0.8221 & 0.8934 & 0.8012 & 0.9792 \\
\hline & & & & & & & & & & \\
\hline & & $\mathrm{L} 2$ & 147 & 0.9558 & 0.8260 & 0.9762 & 0.8260 & 0.8980 & 0.8023 & 0.9800 \\
\hline & & RCA & 150 & 0.9563 & 0.8281 & 0.9771 & 0.8289 & 0.8995 & 0.8056 & 0.9811 \\
\hline & \multirow{5}{*}{ HR-Net } & No & 111 & 0.9530 & 0.8110 & 0.9711 & 0.8124 & 0.8874 & 0.7992 & 0.9782 \\
\hline & & Dropout & 122 & 0.9536 & 0.8179 & 0.9725 & 0.8210 & 0.8919 & 0.7999 & 0.9780 \\
\hline & & & & & & & & & & \\
\hline & & L2 & 118 & 0.9552 & 0.8240 & 0.9743 & 0.8244 & 0.8960 & 0.8003 & 0.9793 \\
\hline & & RCA & 121 & 0.9558 & 0.8266 & 0.9759 & 0.8243 & 0.8982 & 0.8035 & 0.9801 \\
\hline \multirow{15}{*}{ STARE } & & No & 140 & 0.9598 & 0.8231 & 0.9712 & 0.8215 & 0.8941 & 0.8204 & 0.9817 \\
\hline & Single & Dropout & 138 & 0.9615 & 0.8442 & 0.9764 & 0.8342 & 0.9079 & 0.8237 & 0.9831 \\
\hline & branch & $\mathrm{L} 2$ & 151 & 0.9662 & 0.8519 & 0.9798 & 0.8428 & 0.9136 & 0.8273 & 0.9868 \\
\hline & & RCA & 142 & 0.9681 & 0.8598 & 0.9819 & 0.8493 & 0.9188 & 0.8297 & 0.9890 \\
\hline & \multirow{5}{*}{ DBFU-Net } & No & 147 & 0.9527 & 0.8162 & 0.9710 & 0.8213 & 0.8902 & 0.8211 & 0.9814 \\
\hline & & Dropout & 158 & 0.9653 & 0.8498 & 0.9782 & 0.8394 & 0.9117 & 0.8262 & 0.9860 \\
\hline & & & & & & & & & & \\
\hline & & L2 & 164 & 0.9672 & 0.8527 & 0.9799 & 0.9437 & 0.9141 & 0.8301 & 0.9887 \\
\hline & & RCA & 163 & 0.9691 & 0.8612 & 0.9823 & 0.8501 & 0.9198 & 0.8332 & 0.9892 \\
\hline & \multirow{5}{*}{ HR-Net } & No & 136 & 0.9588 & 0.8221 & 0.9704 & 0.8211 & 0.8932 & 0.8199 & 0.9811 \\
\hline & & Dropout & 135 & 0.9606 & 0.8437 & 0.9760 & 0.8332 & 0.9074 & 0.8225 & 0.9823 \\
\hline & & & & & & & & & & \\
\hline & & $\mathrm{L} 2$ & 144 & 0.9659 & 0.8502 & 0.9799 & 0.8414 & 0.9127 & 0.8264 & 0.9854 \\
\hline & & RCA & 147 & 0.9678 & 0.8588 & 0.9802 & 0.8487 & 0.9175 & 0.8290 & 0.9876 \\
\hline & Single & No & 130 & 0.9590 & 0.8391 & 0.9705 & 0.8217 & 0.9024 & 0.8097 & 0.9816 \\
\hline
\end{tabular}




\begin{tabular}{|c|c|c|c|c|c|c|c|c|c|}
\hline \multirow[t]{3}{*}{ branch } & Dropout & 131 & 0.9611 & 0.8441 & 0.9765 & 0.8305 & 0.9079 & 0.8162 & 0.9854 \\
\hline & L2 & 140 & 0.9663 & 0.8489 & 0.9798 & 0.8357 & 0.9120 & 0.8149 & 0.9868 \\
\hline & RCA & 138 & 0.9676 & 0.8507 & 0.9796 & 0.8363 & 0.9129 & 0.8181 & 0.9873 \\
\hline \multirow{5}{*}{ DBFU-Net } & No & 142 & 0.9556 & 0.8372 & 0.9669 & 0.8204 & 0.8997 & 0.8091 & 0.9802 \\
\hline & Dropout & 146 & 0.9635 & 0.8462 & 0.9781 & 0.8312 & 0.9101 & 0.8174 & 0.9861 \\
\hline & & & & & & & & & \\
\hline & L2 & 166 & 0.9677 & 0.8508 & 0.9799 & 0.8364 & 0.9131 & 0.8169 & 0.9869 \\
\hline & RCA & 161 & 0.9682 & 0.8520 & 0.9800 & 0.8375 & 0.9138 & 0.8195 & 0.9879 \\
\hline \multirow{5}{*}{ HR-Net } & No & 125 & 0.9577 & 0.8372 & 0.9698 & 0.8211 & 0.9011 & 0.8086 & 0.9806 \\
\hline & Dropout & 124 & 0.9601 & 0.8429 & 0.9745 & 0.8284 & 0.9063 & 0.8158 & 0.9849 \\
\hline & & & & & & & & & \\
\hline & L2 & 138 & 0.9645 & 0.8470 & 0.9782 & 0.8350 & 0.9102 & 0.8141 & 0.9850 \\
\hline & RCA & 133 & 0.9658 & 0.8499 & 0.9788 & 0.8348 & 0.9121 & 0.8172 & 0.9862 \\
\hline
\end{tabular}

1 


\section{Table 2 (on next page)}

Table 2: Performance of the five models tested on the DRIVE, STARE and CHASE datasets.

The top results for each dataset are marked in bold. DBFU-Net-NH is shorthand for: decoder 2

of DBFU-Net is trained by cross entropy and not using the hard example weighting training strategy. 
1

\begin{tabular}{|c|c|c|c|c|c|c|c|c|c|}
\hline Dataset & Method & Threshold & $\mathrm{ACC}$ & $\mathrm{Se}$ & $\mathrm{Sp}$ & $\mathrm{F} 1$ & G & $\mathrm{MCC}$ & AUC \\
\hline \multirow{5}{*}{ DRIVE } & single branch & 125 & 0.9559 & 0.8265 & 0.9762 & 0.8259 & 0.8982 & 0.8034 & 0.9805 \\
\hline & DBFU-Net-NH & 131 & 0.9554 & 0.8266 & 0.9770 & 0.8264 & 0.8987 & 0.8041 & 0.9801 \\
\hline & Decoder 1 & 122 & 0.9556 & 0.8266 & 0.9771 & 0.8257 & 0.8987 & 0.8033 & 0.9802 \\
\hline & Decoder 2 & 197 & 0.9497 & 0.8534 & 0.9141 & 0.8230 & 0.8832 & 0.8012 & 0.9768 \\
\hline & DBFU-Net & 150 & 0.9563 & 0.8281 & 0.9771 & 0.8289 & 0.8995 & 0.8056 & 0.9811 \\
\hline \multirow{5}{*}{ STARE } & single branch & 142 & 0.9681 & 0.8598 & 0.9819 & 0.8493 & 0.9188 & 0.8297 & 0.9890 \\
\hline & DBFU-Net-NH & 145 & 0.9684 & 0.8599 & 0.9825 & 0.8494 & 0.9192 & 0.8312 & 0.9889 \\
\hline & Decoder 1 & 144 & 0.9679 & 0.8596 & 0.9820 & 0.8492 & 0.9188 & 0.8299 & 0.9887 \\
\hline & Decoder 2 & 206 & 0.9513 & 0.8976 & 0.9284 & 0.8355 & 0.9129 & 0.8203 & 0.9773 \\
\hline & DBFU-Net & 159 & 0.9691 & 0.8612 & 0.9823 & 0.8501 & 0.9198 & 0.8332 & 0.9892 \\
\hline \multirow{5}{*}{ CHASE } & single branch & 138 & 0.9676 & 0.8507 & 0.9796 & 0.8363 & 0.9129 & 0.8181 & 0.9873 \\
\hline & DBFU-Net-NH & 140 & 0.9677 & 0.8510 & 0.9798 & 0.8370 & 0.9131 & 0.8183 & 0.9867 \\
\hline & Decoder 1 & 136 & 0.9673 & 0.8501 & 0.9789 & 0.8358 & 0.9122 & 0.8182 & 0.9868 \\
\hline & Decoder 2 & 215 & 0.9509 & 0.8937 & 0.9204 & 0.8211 & 0.9070 & 0.8099 & 0.9748 \\
\hline & DBFU-Net & 161 & 0.9682 & 0.8520 & 0.9800 & 0.8375 & 0.9138 & 0.8195 & 0.9879 \\
\hline
\end{tabular}

2 


\section{Table 3 (on next page)}

Table 3: Performance of the seven models tested on the DRIVE, STARE and CHASE dataset. The top results for each dataset are marked in bold. SB-F represents a single branch model trained by focal loss, SB-D represents a single branch model trained by dice 
1

\begin{tabular}{|c|c|c|c|c|c|c|c|c|c|}
\hline Dataset & Method & Threshold & $\mathrm{ACC}$ & $\mathrm{Se}$ & $\mathrm{Sp}$ & $\mathrm{F} 1$ & G & $\mathrm{MCC}$ & AUC \\
\hline \multirow{3}{*}{ DRIVE } & DBFU-Net-D & 141 & 0.9557 & 0.8259 & 0.9769 & 0.8270 & 0.8982 & 0.8038 & 0.9802 \\
\hline & SB-D & 147 & 0.9542 & 0.8347 & 0.9568 & 0.8239 & 0.8937 & 0.8013 & 0.9789 \\
\hline & Decoder 2 & 197 & 0.9497 & 0.8534 & 0.9141 & 0.8230 & 0.8832 & 0.8012 & 0.9768 \\
\hline \multirow{4}{*}{ STARE } & DBFU-Net-F & 169 & 0.9679 & 0.8603 & 0.9808 & 0.8495 & 0.9185 & 0.8321 & 0.9891 \\
\hline & DBFU-Net-D & 152 & 0.9689 & 0.8591 & 0.9810 & 0.8490 & 0.9180 & 0.8316 & 0.9890 \\
\hline & SB-F & 174 & 0.9544 & 0.8839 & 0.9325 & 0.8402 & 0.9079 & 0.8227 & 0.9817 \\
\hline & SB-D & 155 & 0.9596 & 0.8783 & 0.9457 & 0.8429 & 0.9114 & 0.8275 & 0.9877 \\
\hline \multirow{6}{*}{ CHASE } & DBFU-Net-D & 188 & 0.9672 & 0.8501 & 0.9804 & 0.8358 & 0.9129 & 0.8181 & 0.9869 \\
\hline & SB-F & 195 & 0.9518 & 0.8902 & 0.9299 & 0.8189 & 0.9098 & 0.8003 & 0.9716 \\
\hline & & & & & & & & & \\
\hline & SB-D & 176 & 0.9588 & 0.8740 & 0.9411 & 0.8245 & 0.9069 & 0.8107 & 0.9823 \\
\hline & Decoder 2 & 215 & 0.9509 & 0.8937 & 0.9204 & 0.8211 & 0.9070 & 0.8099 & 0.9748 \\
\hline & DBFU-Net & 161 & 0.9682 & 0.8520 & 0.9800 & 0.8375 & 0.9138 & 0.8195 & 0.9879 \\
\hline
\end{tabular}




\section{Table 4 (on next page)}

Table 4: Performance of the different models tested on the DRIVE dataset. The top results for each dataset are marked in bold. "-" that the values are not given in these papers. 
1

\begin{tabular}{|c|c|c|c|c|c|c|c|c|}
\hline Type & Method & $\mathrm{ACC}$ & $\mathrm{Se}$ & $\mathrm{Sp}$ & $\mathrm{F} 1$ & G & $\mathrm{MCC}$ & AUC \\
\hline \multirow{2}{*}{ unsupervised } & Neto et al.[10] & 0.8718 & 0.7806 & 0.9629 & - & 0.867 & - & - \\
\hline & Zhang et al.[11] & 0.959 & 0.745 & 0.976 & - & 0.8527 & - & 0.861 \\
\hline \multirow{11}{*}{ supervised } & Yang et al.[15] & 0.9694 & 0.7762 & 0.9835 & - & 0.8737 & - & - \\
\hline & Leopold et al.[16] & 0.9106 & 0.6963 & 0.9573 & 0.7382 & 0.8159 & 0.6820 & - \\
\hline & Kromm et al.[18] & 0.9547 & 0.7651 & 0.9818 & 0.8192 & 0.8667 & - & 0.9750 \\
\hline & Ribeiro et al.[19] & 0.9569 & 0.7880 & 0.9819 & - & 0.8796 & - & - \\
\hline & Adarsh et al.[20] & 0.9563 & 0.7979 & 0.9794 & 0.8227 & 0.8840 & - & 0.9795 \\
\hline & & & & & & & & \\
\hline & Guo et al.[21] & 0.9561 & 0.7891 & 0.9804 & 0.8249 & 0.8796 & 0.7964 & 0.9806 \\
\hline & Palanivel et al.[13] & 0.9480 & 0.7375 & 0.9788 & - & 0.8496 & - & 0.9590 \\
\hline & Zhao et al.[23] & - & 0.8329 & 0.9767 & 0.8229 & 0.9019 & - & - \\
\hline & Li et al.[34] & 0.9339 & 0.8063 & 0.9529 & - & 0.8761 & - & - \\
\hline & DBFU-Net & 0.9563 & 0.8281 & 0.9771 & 0.8289 & 0.8995 & 0.8056 & 0.9811 \\
\hline
\end{tabular}




\section{Table 5 (on next page)}

Table 5: Performance of the different models tested on the STARE dataset. The top results for each dataset are marked in bold. "-" that the values are not given in these papers. 


\begin{tabular}{|c|c|c|c|c|c|c|c|c|}
\hline Type & Method & $\mathrm{ACC}$ & $\mathrm{Se}$ & $\mathrm{Sp}$ & $\mathrm{F} 1$ & G & MCC & AUC \\
\hline \multirow[t]{2}{*}{ non-learning } & Sazak et al.[9] & 0.962 & 0.730 & 0.979 & - & 0.8453 & - & - \\
\hline & Neto et al.[10] & 0.8894 & 0.8344 & 0.9443 & - & 0.8877 & - & - \\
\hline & Zhang et al.[11] & 0.951 & 0.799 & 0.962 & - & 0.8767 & - & \\
\hline \multirow{9}{*}{ supervised } & Yang et al.[15] & 0.9537 & 0.7721 & 0.9885 & - & 0.8736 & - & - \\
\hline & Leopold et al.[16] & 0.9045 & 0.6433 & 0.9472 & 0.6465 & 0.7797 & 0.5960 & - \\
\hline & Li et al.[17] & 0.9620 & 0.7798 & 0.9822 & 0.8037 & 0.8752 & - & 0.9791 \\
\hline & Guo et al.[21] & 0.9674 & 0.8212 & 0.9843 & 0.8421 & 0.8991 & 0.8221 & 0.9859 \\
\hline & Yan et al.[22] & 0.9638 & 0.7735 & 0.9857 & - & 0.8732 & - & 0.9872 \\
\hline & Palanivel et al.[13] & 0.9542 & 0.7484 & 0.9780 & - & 0.8555 & - & 0.9711 \\
\hline & Zhao et al.[23] & - & 0.6433 & 0.9472 & 0.6465 & 0.7806 & - & - \\
\hline & Li et al.[34] & 0.9422 & 0.8394 & 0.9536 & - & 0.8932 & - & - \\
\hline & DBFU-Net & 0.9691 & 0.8612 & 0.9823 & 0.8501 & 0.9198 & 0.8332 & 0.9892 \\
\hline
\end{tabular}




\section{Table 6(on next page)}

Table 6: Performance of the different models tested on the CHASE dataset. The top results for each dataset are marked in bold. "-" that the values are not given in these papers. 
1

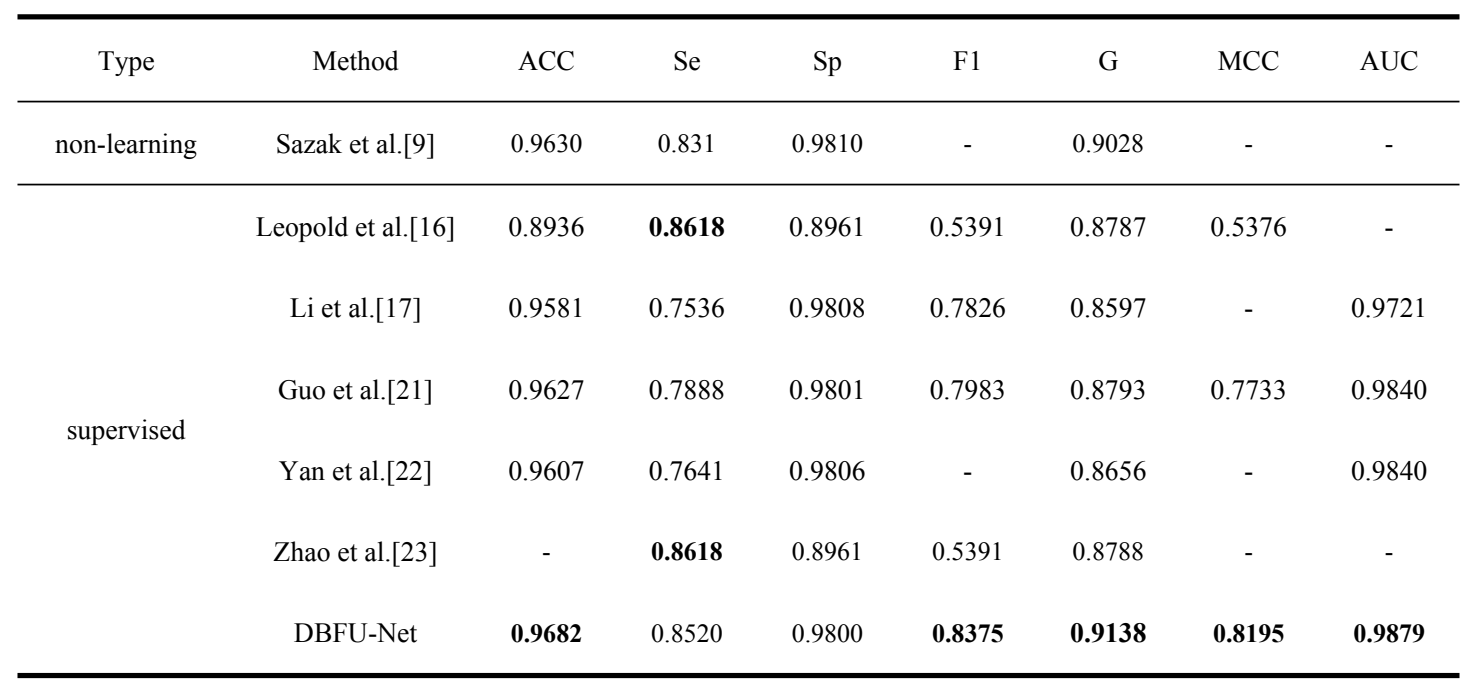

2 


\section{Table 7 (on next page)}

Table 7: Model performance measures from cross-training.

Our result from model that without fine tuning. The top results for each dataset are marked in bold. DBFU-Net-Dropout represents a DBFU-Net trained by Dropout]DBFU-Net-L2 represents a DBFU-Net trained by L2 Regularization. "-" that the values are not given in these papers. 


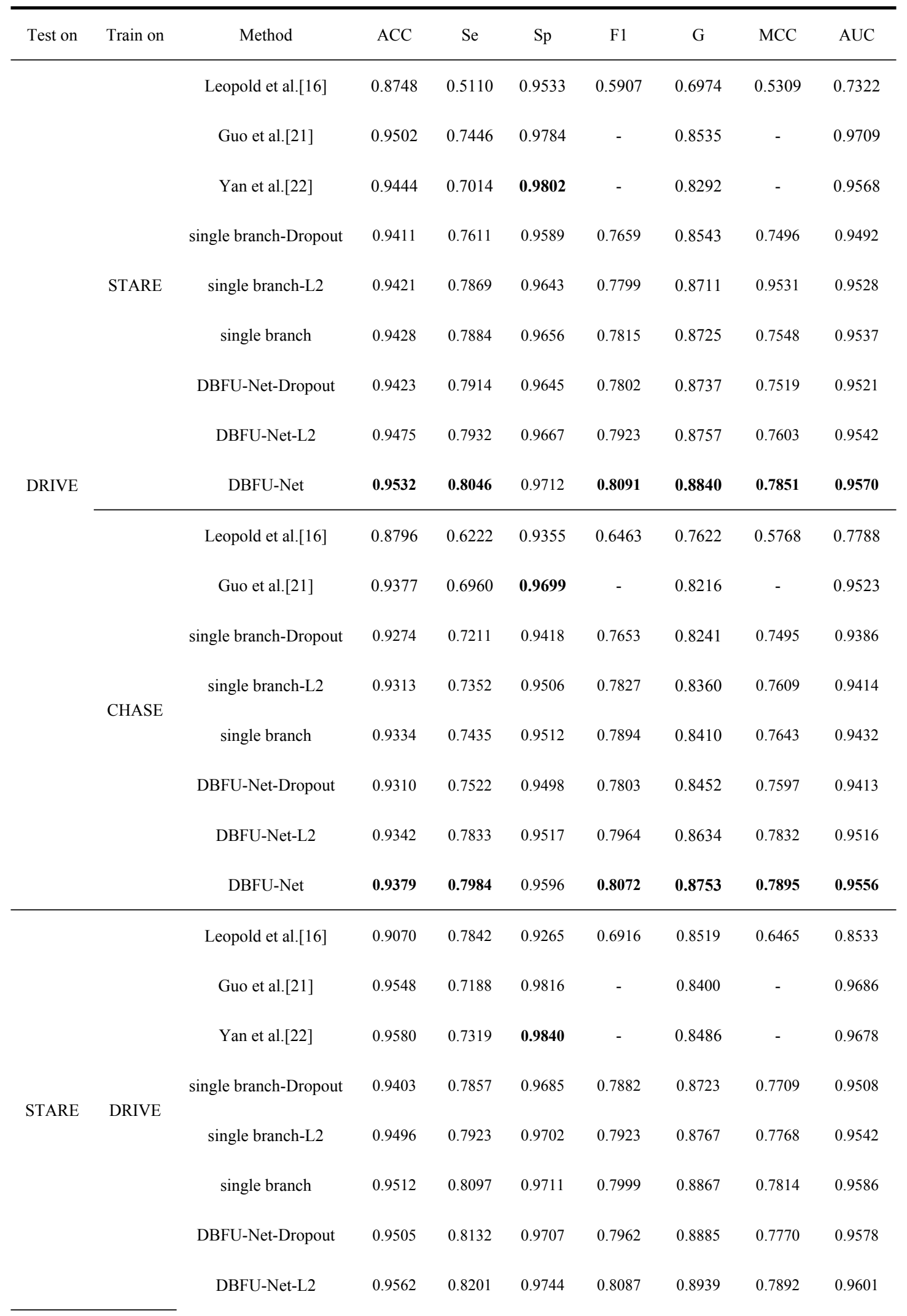




\begin{tabular}{|c|c|c|c|c|c|c|c|c|c|}
\hline & & DBFU-Net & 0.9601 & 0.8235 & 0.9761 & 0.8121 & 0.8966 & 0.7935 & 0.9701 \\
\hline & \multirow{9}{*}{ CHASE } & Leopold et al.[16] & 0.8771 & 0.6973 & 0.9062 & 0.6057 & 0.7941 & 0.5441 & 0.8017 \\
\hline & & Guo et al.[21] & 0.9501 & 0.6799 & 0.9808 & - & 0.8166 & - & 0.9686 \\
\hline & & single branch-Dropout & 0.9413 & 0.7724 & 0.9597 & 0.7743 & 0.8610 & 0.7522 & 0.9502 \\
\hline & & single branch-L2 & 0.9485 & 0.7813 & 0.9624 & 0.7862 & 0.8671 & 0.7597 & 0.9563 \\
\hline & & & & & & & & & \\
\hline & & single branch & 0.9502 & 0.7845 & 0.9689 & 0.7894 & 0.8718 & 0.7610 & 0.9598 \\
\hline & & DBFU-Net-Dropout & 0.9495 & 0.7902 & 0.9603 & 0.7884 & 0.8711 & 0.7603 & 0.9592 \\
\hline & & DBFU-Net-L2 & 0.9537 & 0.7993 & 0.9677 & 0.7921 & 0.8795 & 0.7654 & 0.9615 \\
\hline & & DBFU-Net & 0.9565 & 0.8045 & 0.9704 & 0.8017 & 0.8836 & 0.7756 & 0.9691 \\
\hline \multirow{18}{*}{ CHASE } & \multirow{9}{*}{ DRIVE } & Leopold et al.[16] & 0.8901 & 0.9038 & 0.8891 & 0.5416 & 0.8963 & 0.5480 & 0.8964 \\
\hline & & Guo et al.[21] & 0.9411 & 0.6726 & 0.9710 & - & 0.8081 & - & 0.9511 \\
\hline & & single branch-Dropout & 0.9302 & 0.8299 & 0.9204 & 0.8048 & 0.8740 & 0.7842 & 0.9478 \\
\hline & & single branch-L2 & 0.9375 & 0.8315 & 0.9255 & 0.8097 & 0.8772 & 0.7891 & 0.9486 \\
\hline & & & & & & & & & \\
\hline & & single branch & 0.9387 & 0.8312 & 0.9298 & 0.8102 & 0.8791 & 0.7903 & 0.9509 \\
\hline & & DBFU-Net-Dropout & 0.9382 & 0.8347 & 0.9287 & 0.8104 & 0.8804 & 0.7898 & 0.9507 \\
\hline & & DBFU-Net-L2 & 0.9425 & 0.8386 & 0.9304 & 0.8148 & 0.8833 & 0.7962 & 0.9568 \\
\hline & & DBFU-Net & 0.9457 & 0.8404 & 0.9312 & 0.8198 & 0.8846 & 0.7991 & 0.9638 \\
\hline & \multirow{9}{*}{ STARE } & Leopold et al.[16] & 0.9173 & 0.7525 & 0.9302 & 0.5688 & 0.8365 & 0.5475 & 0.8413 \\
\hline & & Guo et al.[21] & 0.9441 & 0.6980 & 0.9715 & - & 0.8235 & - & 0.9565 \\
\hline & & single branch-Dropout & 0.9327 & 0.7962 & 0.9439 & 0.8084 & 0.8669 & 0.7808 & 0.9571 \\
\hline & & single branch-L2 & 0.9358 & 0.8013 & 0.9482 & 0.8102 & 0.8717 & 0.7843 & 0.9593 \\
\hline & & & & & & & & & \\
\hline & & single branch & 0.9395 & 0.8024 & 0.9497 & 0.8106 & 0.8729 & 0.7865 & 0.9602 \\
\hline & & DBFU-Net-Dropout & 0.9364 & 0.8089 & 0.9473 & 0.8104 & 0.8754 & 0.7853 & 0.9611 \\
\hline & & DBFU-Net-L2 & 0.9411 & 0.8102 & 0.9501 & 0.8155 & 0.8774 & 0.7912 & 0.9645 \\
\hline & & DBFU-Net & 0.9466 & 0.8141 & 0.9539 & 0.8173 & 0.8812 & 0.7973 & 0.9692 \\
\hline
\end{tabular}


Figure 1

Fig.1 Hard example mask extraction via probability range.

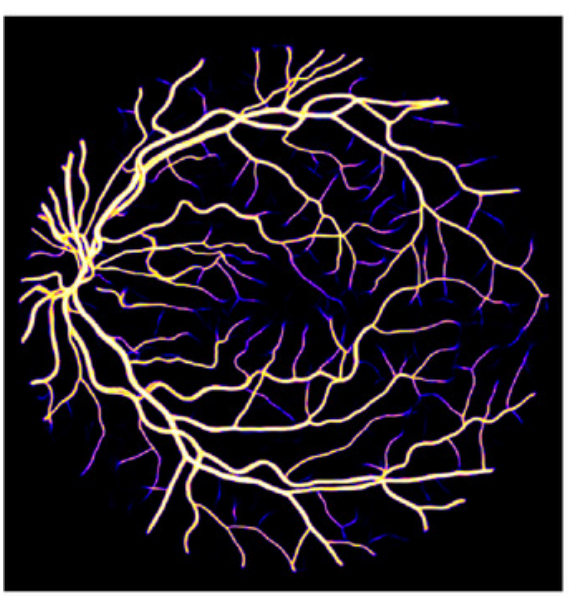

Extract hard example mask by probability range: $[0.2,0.7]$

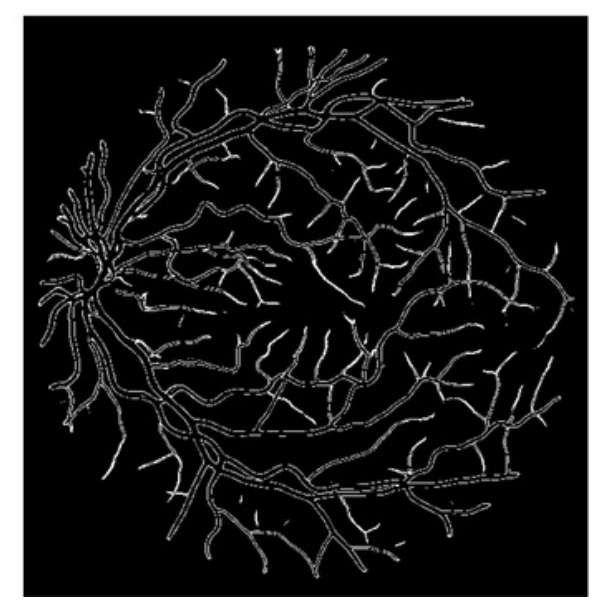




\section{Figure 2}

Fig.2 Hard example masks extracted by morphology.

(a) original label; (b) positive examples extracted by (a) after eroding; (c) hard examples of positive examples obtained by (a)-(b) that contain the edges of large vessels and thin vessels; (d) extracted via the dilated (c) and can further obtain hard examples among the negative samples, e.g., the edges of large vessels and the area near the thin vessels. However, the operation brought redundant non-hard positive examples; (e) is final hard example mask, that extract by (d)-(b), can remove the redundant non-hard positive examples.

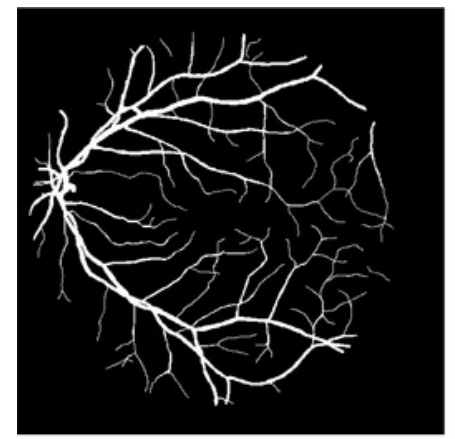

(a)

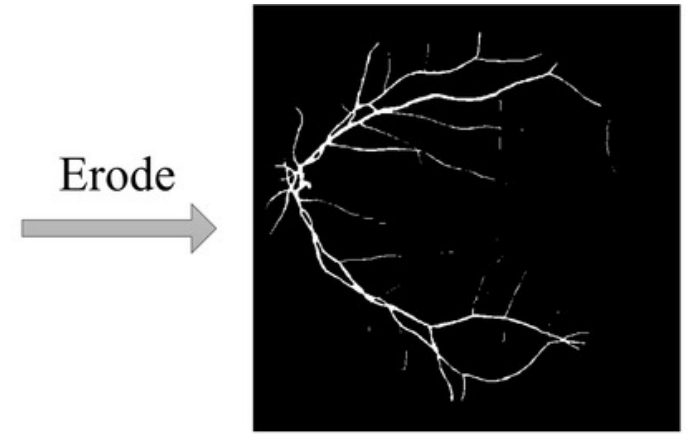

(b)

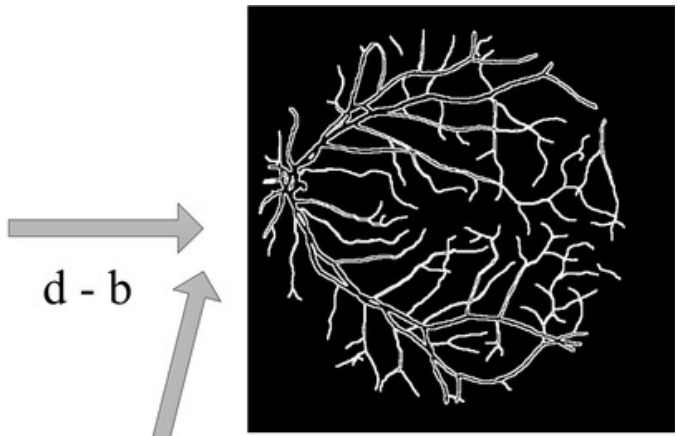

(e)

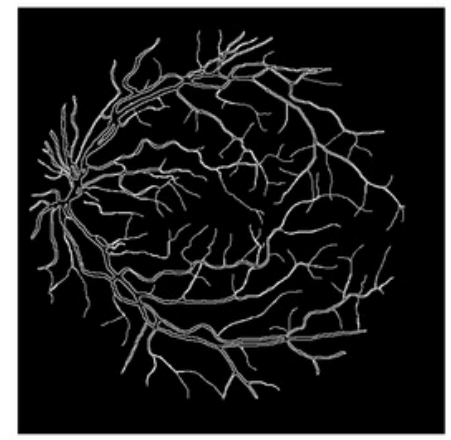

(c)

\section{Dilate}

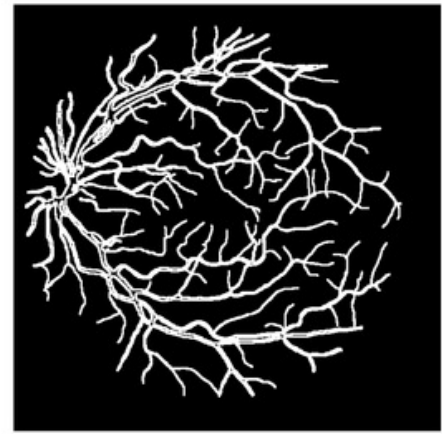

(d) 


\section{Figure 3}

Fig.3 The details of hard example masks are extracted by morphology-based method.

The white part is the label of the fundus image, and the red mask is a hard example mask extracted by the proposed method.

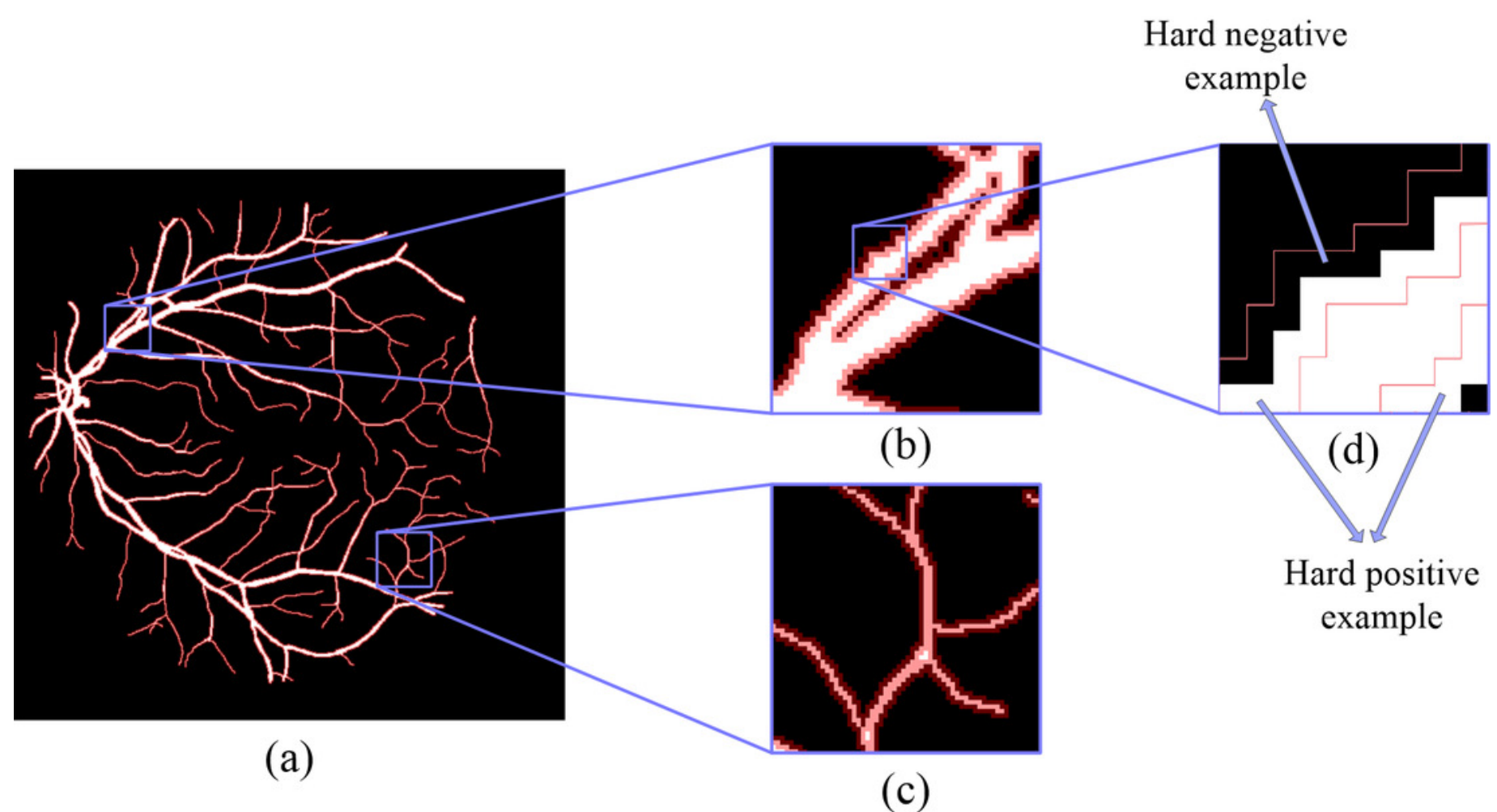


Figure 4

Fig.4 Random channel attention mechanism.

Random weight values will be weighting to each channel of feature maps.

Radom Weight

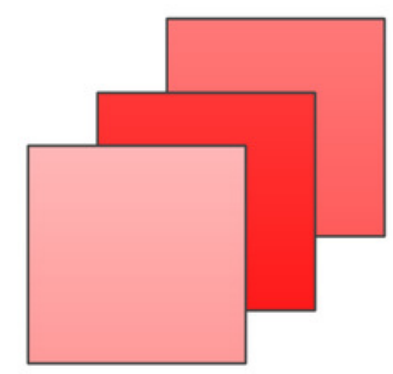

Channel-wise Multiply
High Weight Value

\section{Low Weight Value}

Feature Maps 
Figure 5

Fig.5 The architecture of the proposed DBFU-Net.

DBFU-Net is composed of four parts: an encoder, the first decoder, the second decoder and the fusion layer.

Pixel-wise weighting to cross entropy

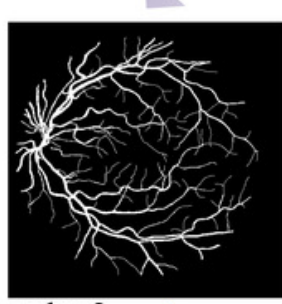

Decoder 2 output

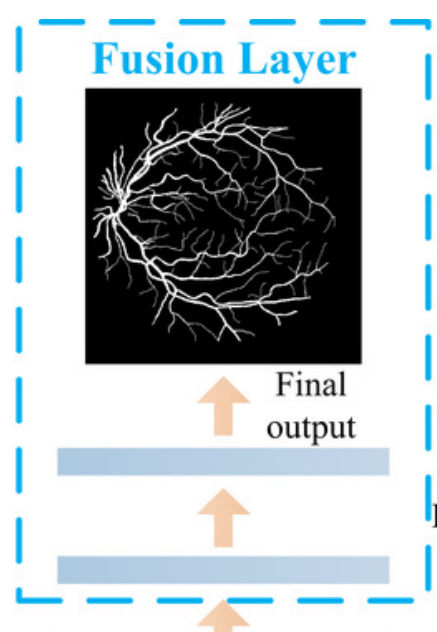

Feature combine with two decoders

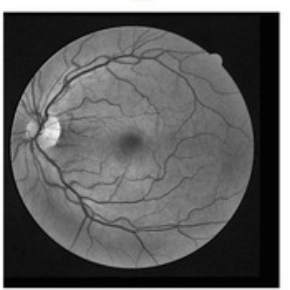

Input image
Res Block

Down Sample

Up Sample

Convolution + Batch Normalization + Activation

Concatenation

Training by the cross entropy

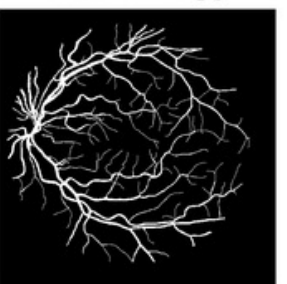

Decoder 1 output

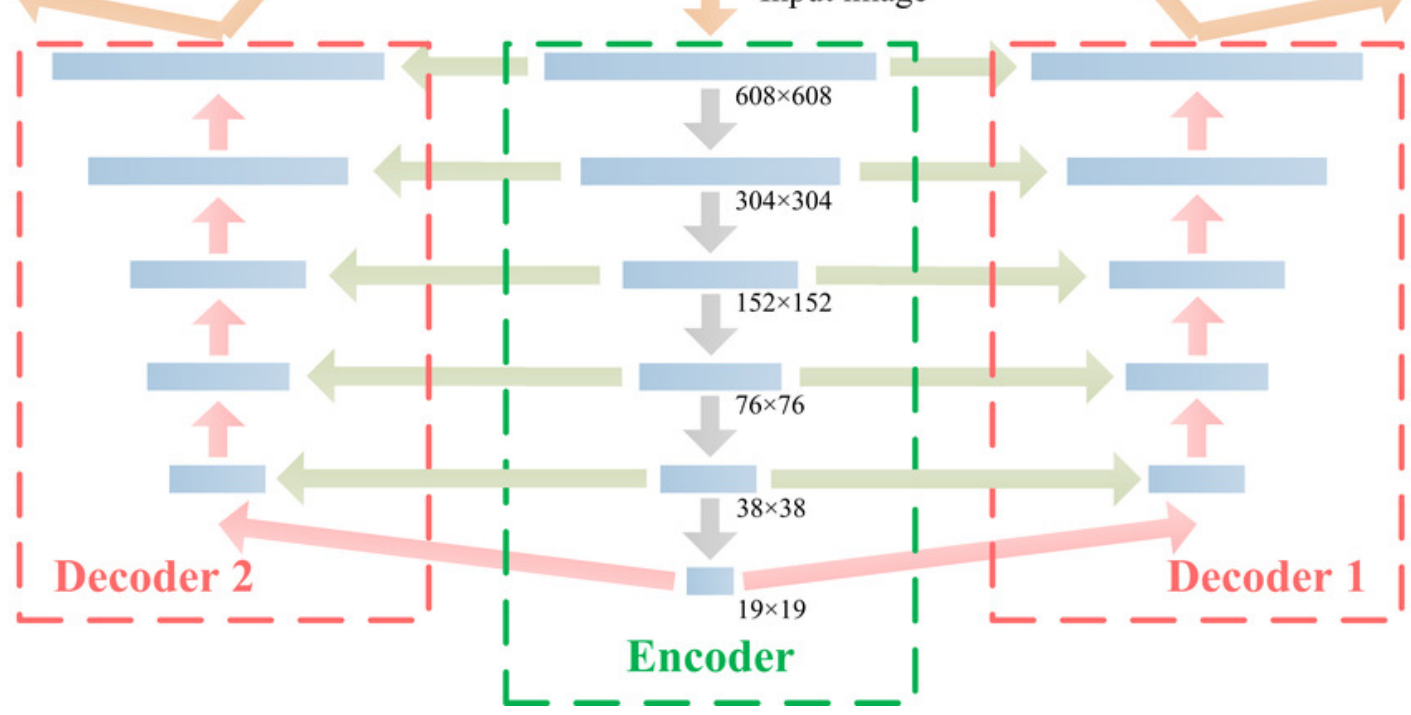


Figure 6

Fig.6 The architecture of Res-block in DBFU-Net.

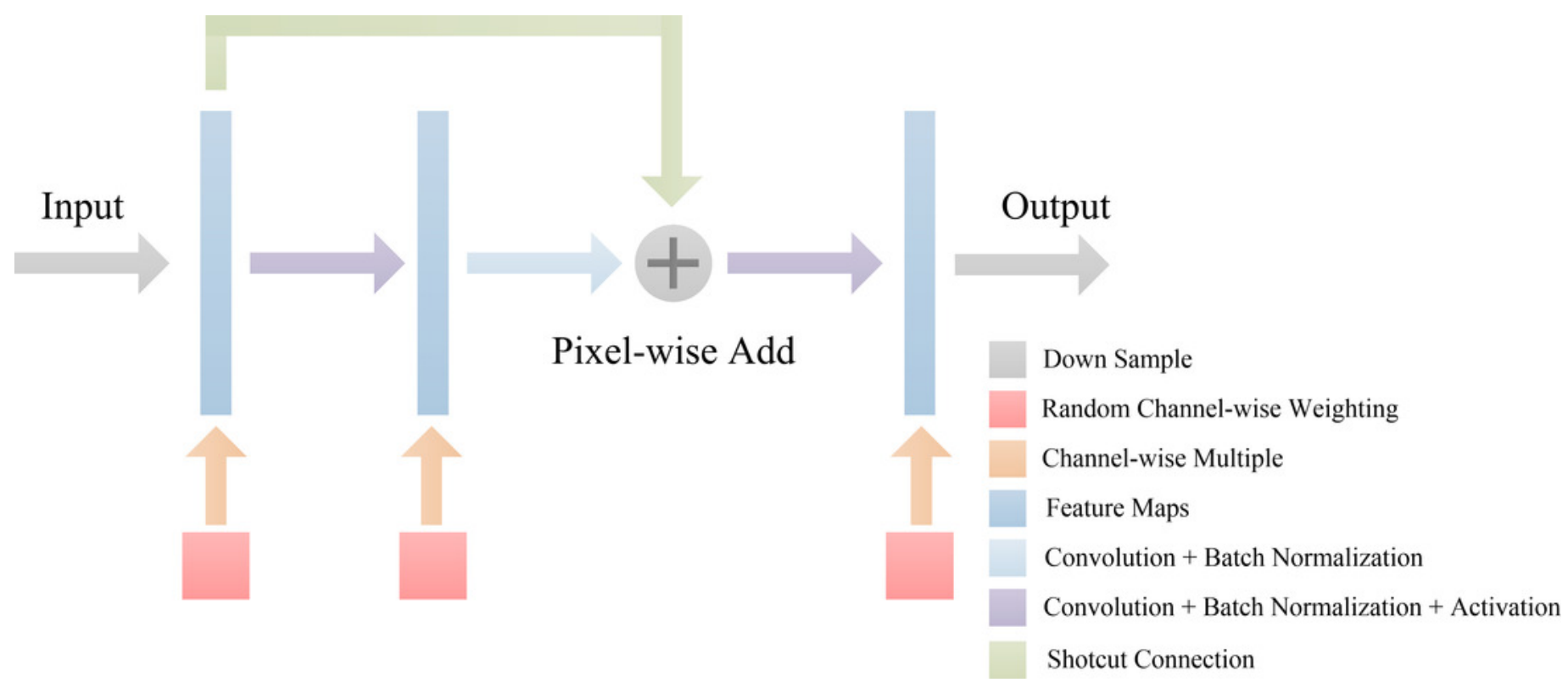


Figure 7

Fig.7 Augmented data by random elastic deformation.

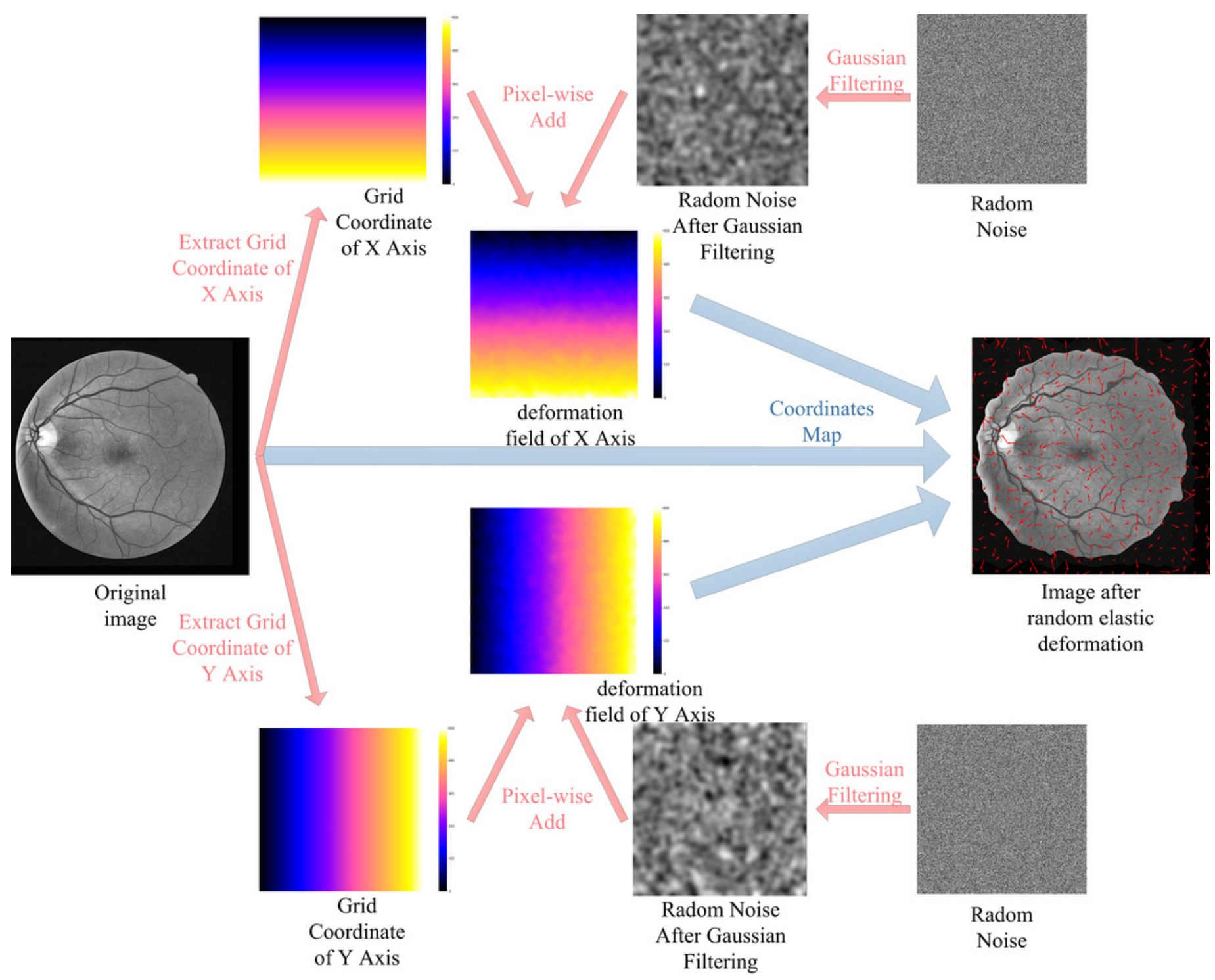


Figure 8

Fig.8 Sample fundus images in DRIVE(rows (I)), STARE(rows (II)) and CHASE(rows (III)) dataset.

(a) fundus image; (b) label of (a); (c) FOV mask of (a).

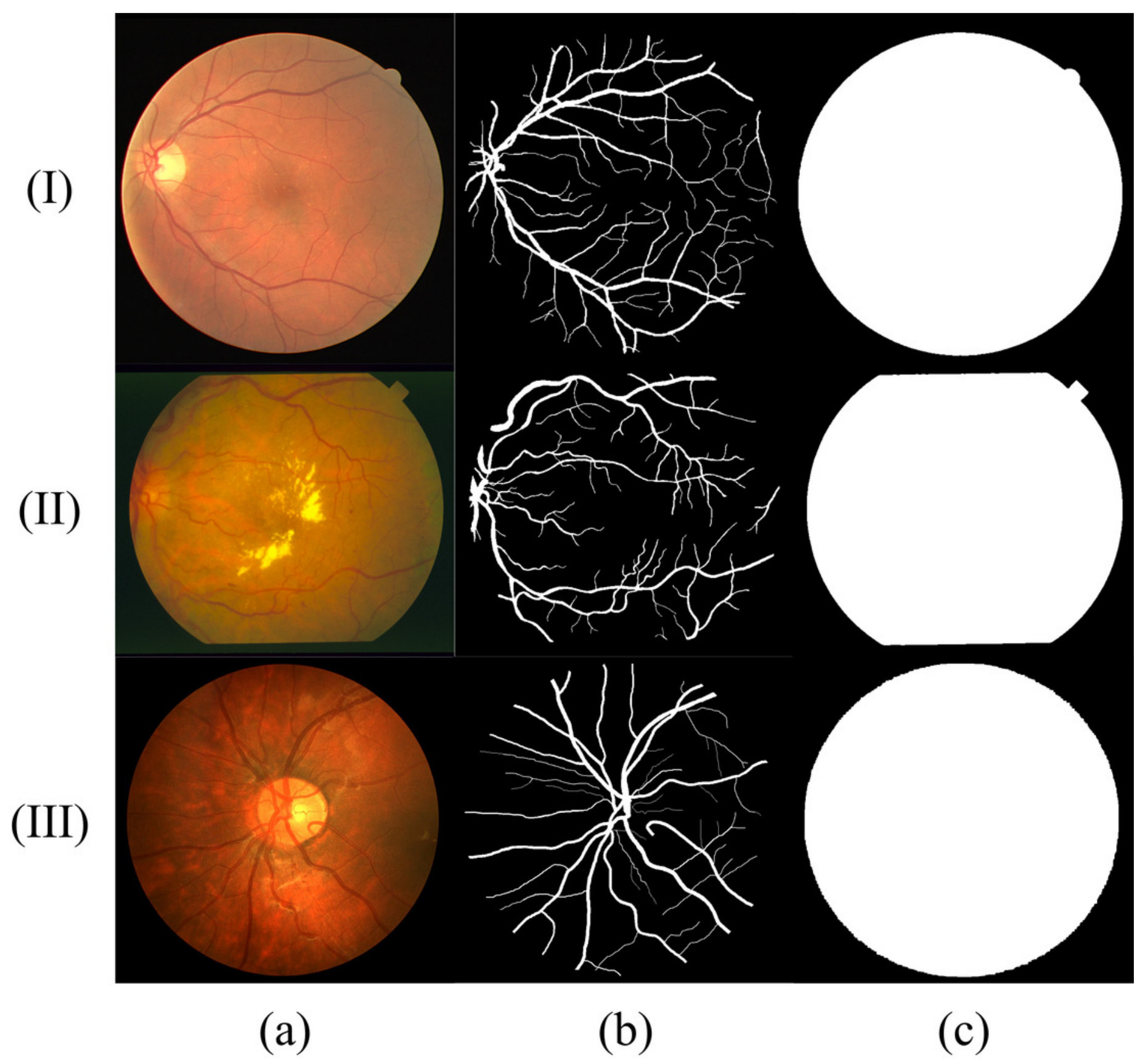


Figure 9

Fig.9 Training loss curves of the model trained by different regularization methods using DRIVE, STARE and CHASE datasets.

The columns of (a), (b), and (c) represent the training process performance of the model using the DRIVE, STARE, and CHASE data sets, respectively. Row (I) is the training loss curve of single branch model for each dataset; row (II) is the training loss curve of DBFU-Net for each dataset; row (III) is the training loss curve of HR-Net for each dataset.
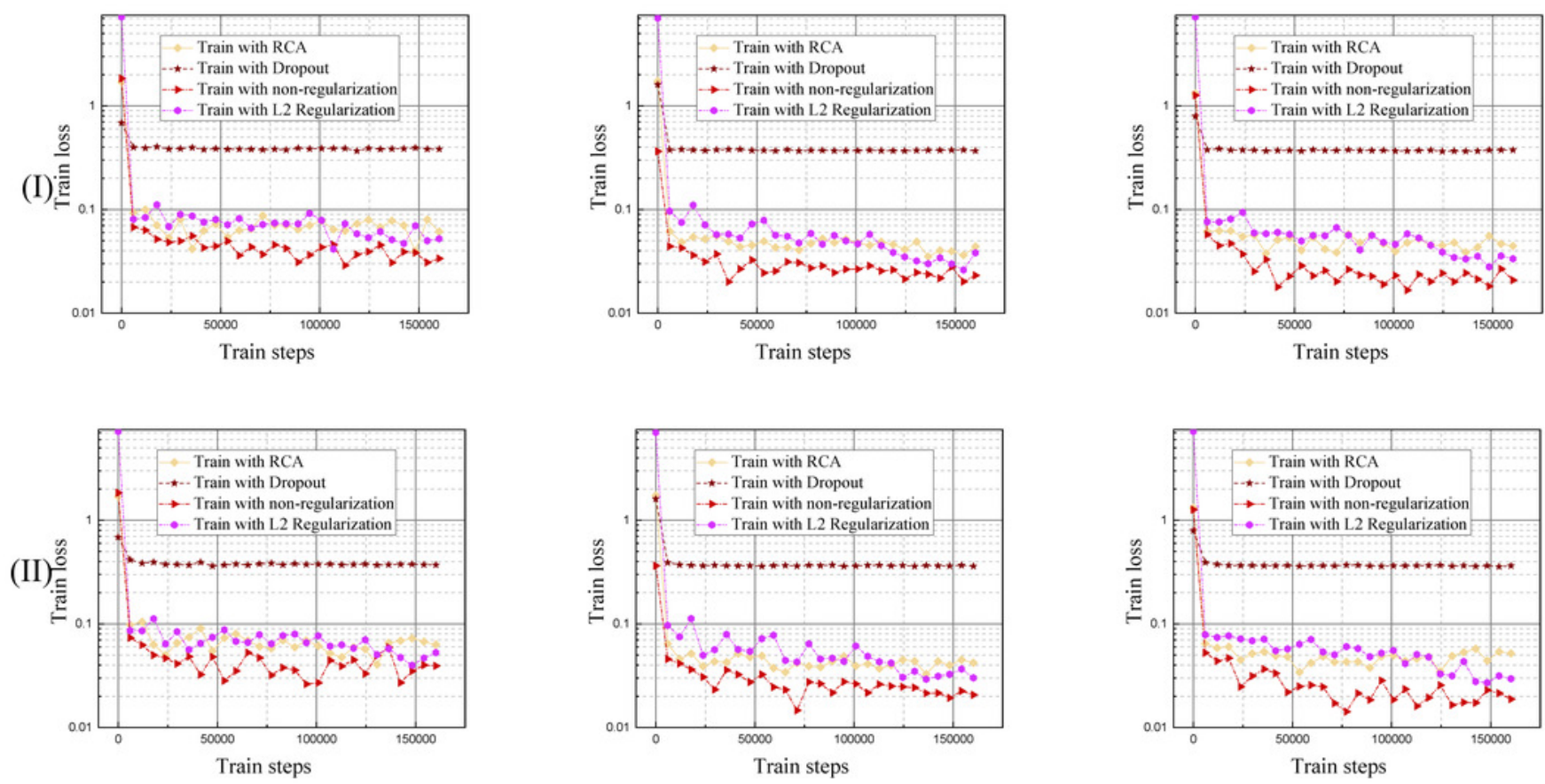

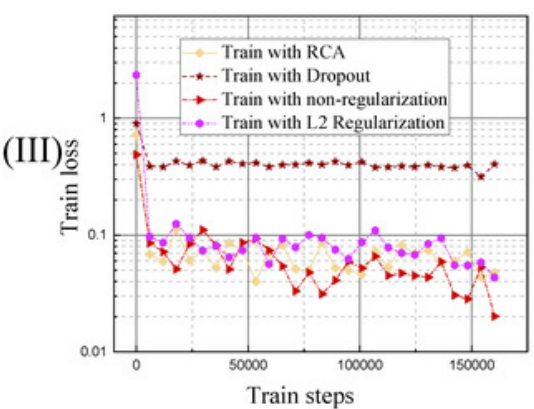

(a)

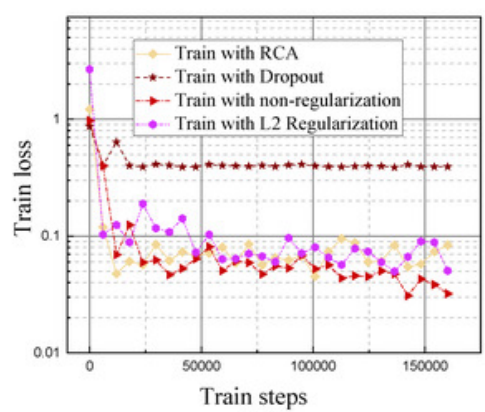

(b)

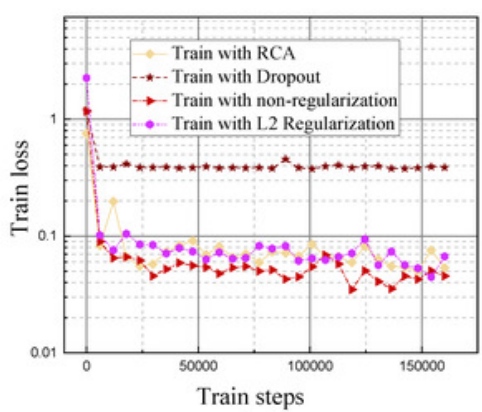

(c) 
Figure 10

Fig.10 Validation loss curves of the model trained by different regularization methods using DRIVE, STARE and CHASE datasets.

The columns of (a), (b), and (c) represent the training process performance of the model for the DRIVE, STARE, and CHASE data sets, respectively. The row (I) is the validation loss curve of a single branch model for each dataset; row (II) is the validation loss curve of DBFU-Net for each dataset; row (III) is the validation loss curve of HR-Net for each dataset.
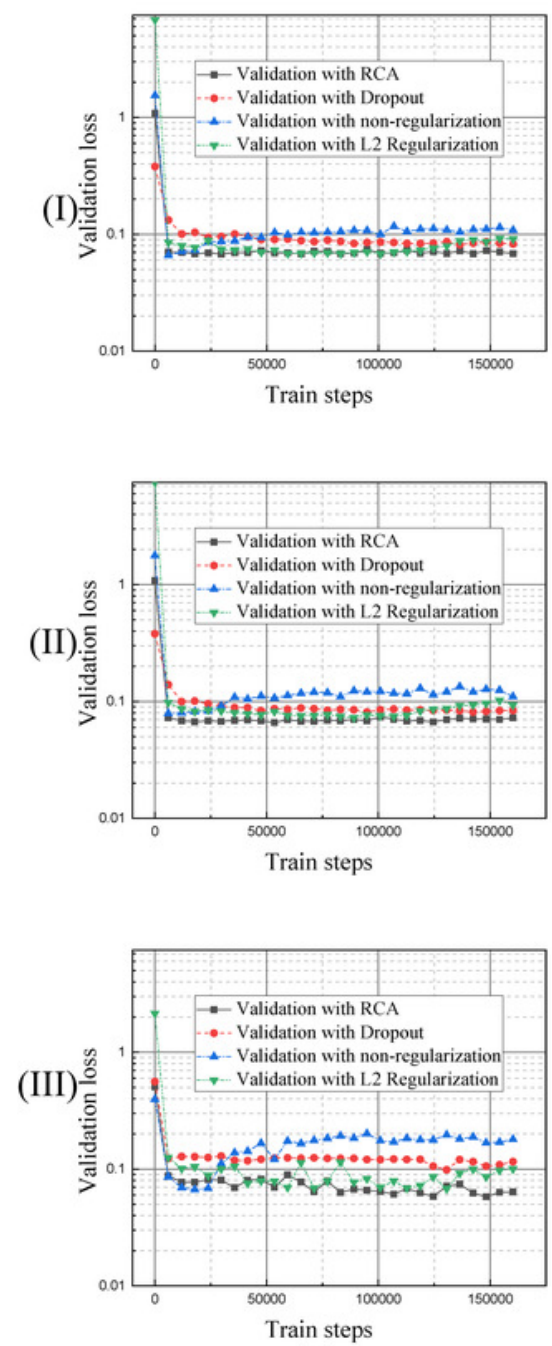

(a)
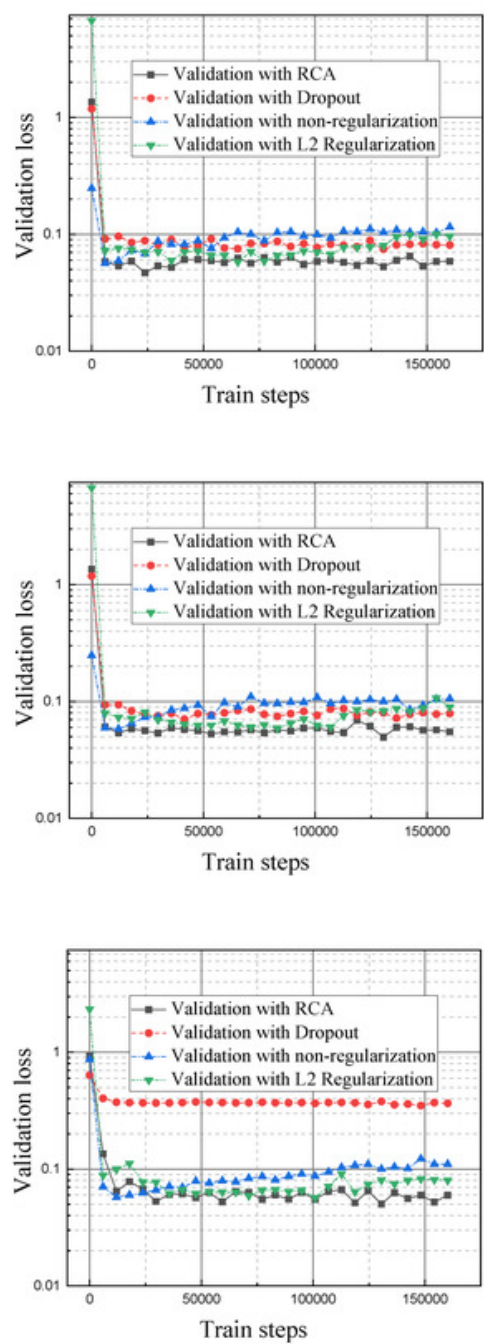

(b)
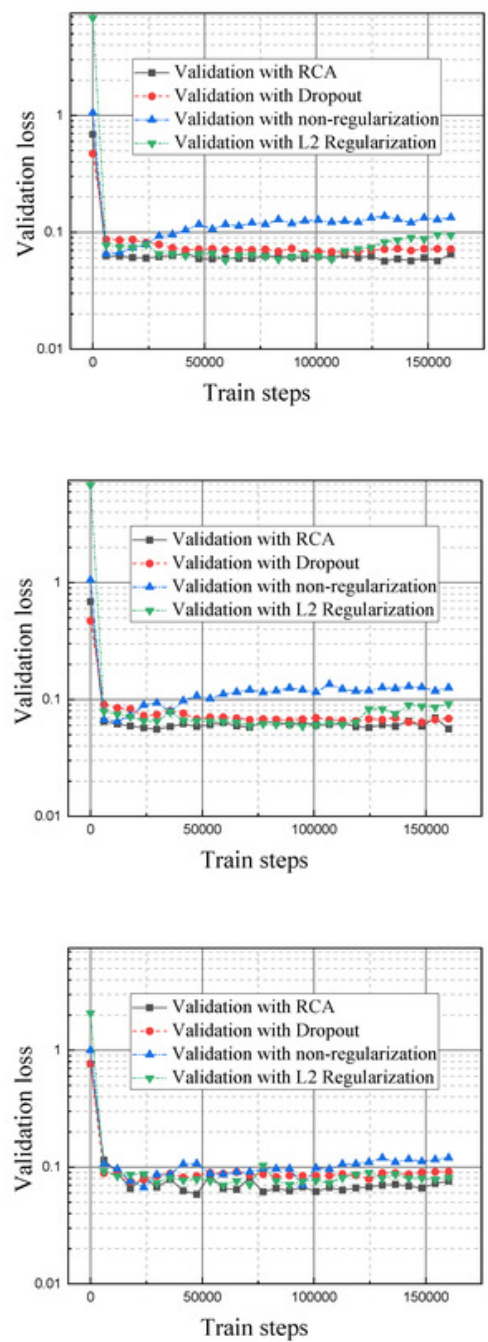

(c) 


\section{Figure 11}

Fig.11 Performance of DBFU-Net given three datasets.

Each row of figure represents the performance of DBFU-Net on the DRIVE ((A) - (E)), STARE $((F)-(J))$, and CHASE $((K)-(O))$ data sets, respectively. Each column of figure represents the original fundus images $((A)-(K))$; the label of corresponding original fundus images ((B) $(L))$; the outputs of first decoder ((C) - (M)); the outputs of second decoder ((D) - (N)); the outputs of fusion layer and that are final segmentation probability distribution maps ((E) (Q)), respectively.

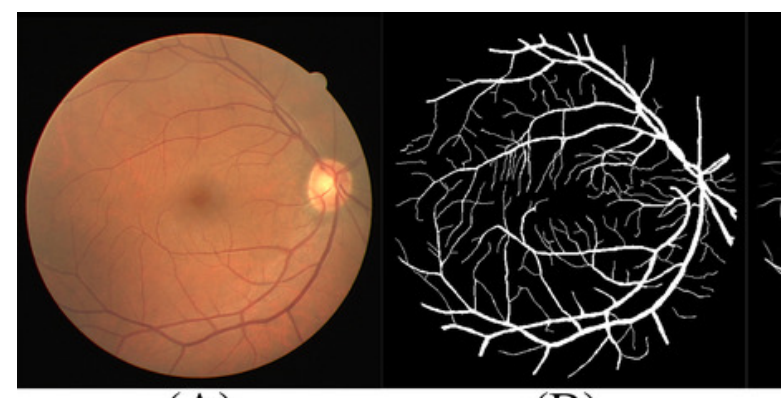

(A)

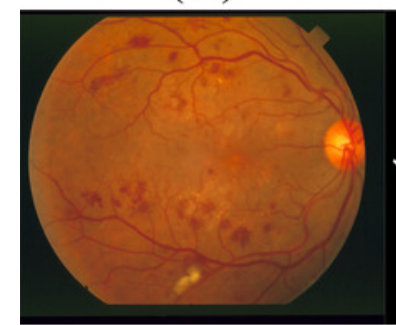

(F)

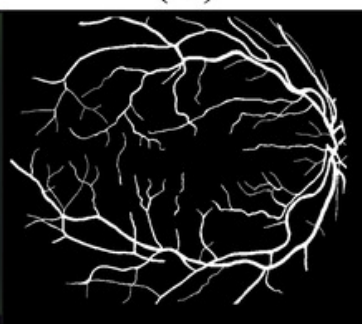

(G)

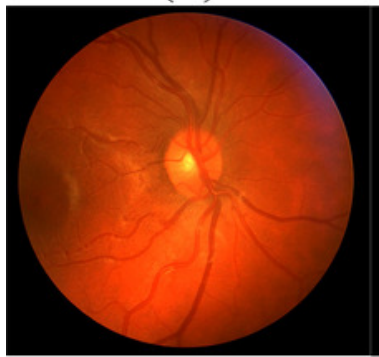

$(\mathrm{K})$

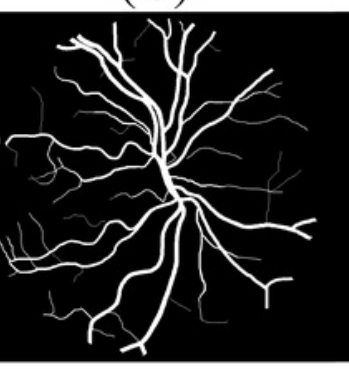

(L)

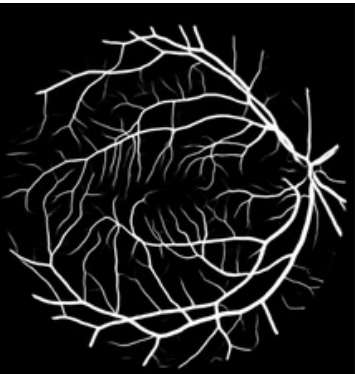

(C)

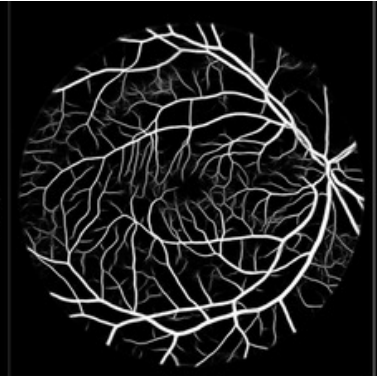

(D)

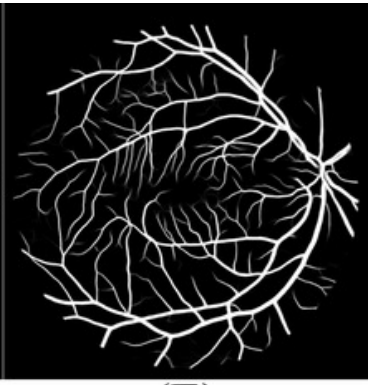

(E)

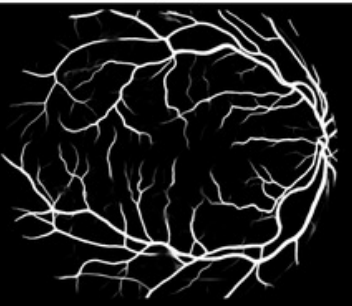

(H)

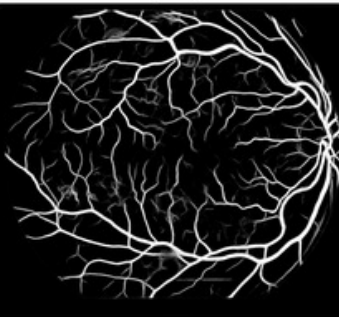

(I)

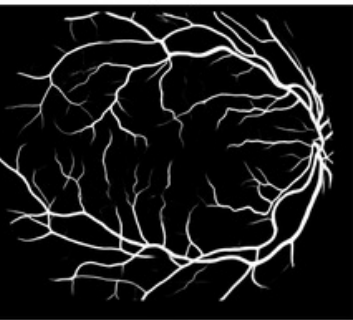

(J)

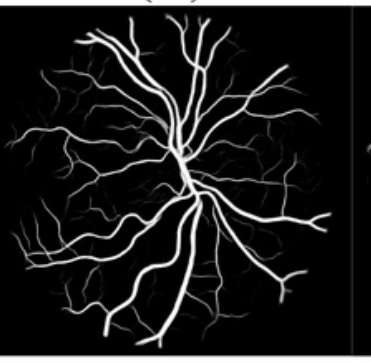

(M)

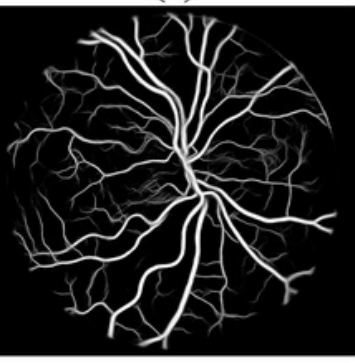

(N)

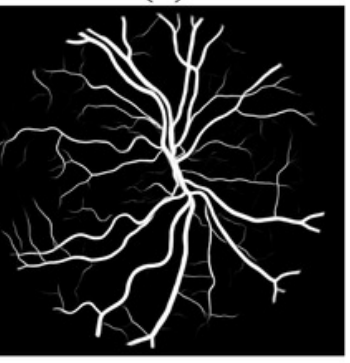

(O) 


\section{Figure 12}

Fig.12 The contrast of the final fusion output probability map and output of double decoders.

Each row of figure represents the DRIVE ((A) - (F)), STARE ((G) - (L)), and CHASE ((M) - (R)) data sets, respectively. Each column of figure represents the original fundus images ((A) $(M))$; the detail of the regions ((B) - $(N)$ ) that have been selected in corresponding original fundus images; the labels of corresponding regions ((C) - (O)); the final segmentation results generated by DBFU-Net ((D) - (P)); the segmentation results of second decoder ((E) - $(Q))$; the segmentation results of the first decoder ((F) - $(R))$. In the final segmentation results, the segmentation results of second decoder, and the segmentation results of the first decoder, we used green, blue, red and black to represent TP, FP, FN, TN, respectively.

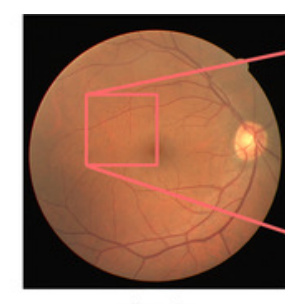

(A)

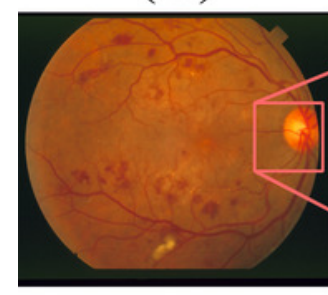

(G)

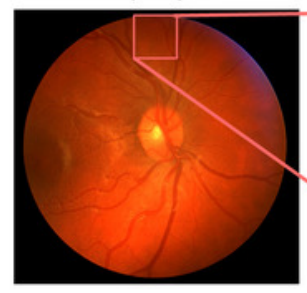

(M)

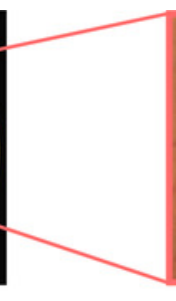

.

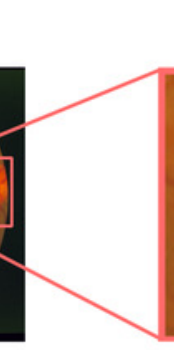

(B)

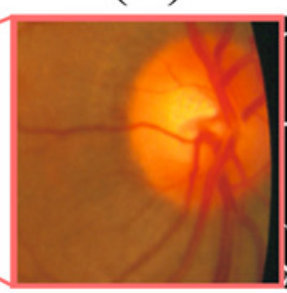

(H)
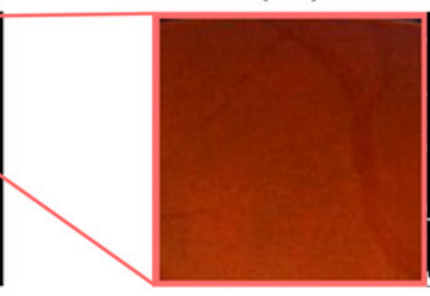

(N)

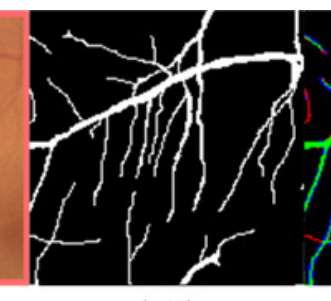

(C)

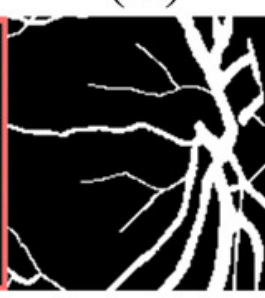

(I)

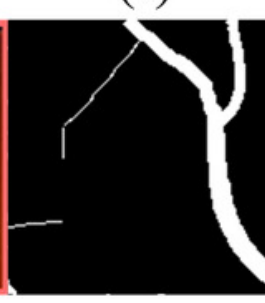

(O)

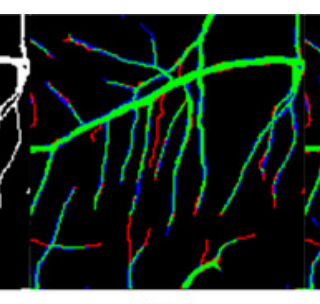

(D)

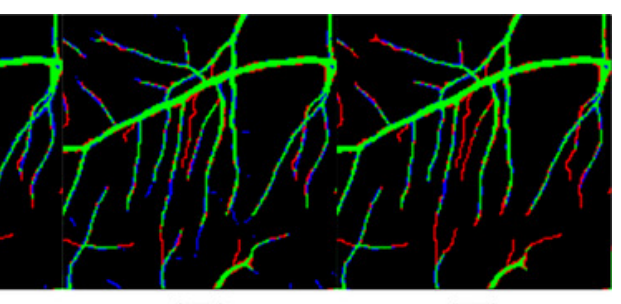

(E)

(F)

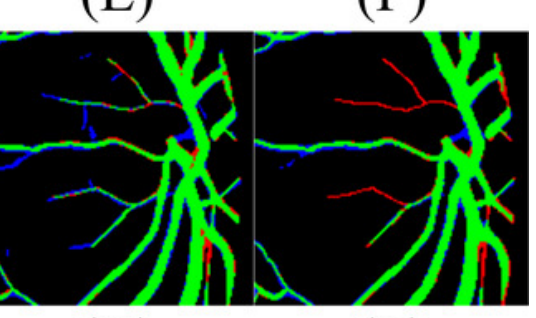

(J)

(K)

(L)

(K)

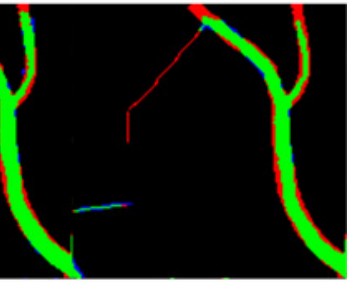

(P)

(Q)

(R) 


\section{Figure 13}

Fig.13. The performances of different methods in hard examples.

Each row of figure shows detection of optic discs $((A)-(F))$, small vascular $((G)-(L))$, and lesion areas $((M)-(R))$, respectively. Each column of figure shows the original images $((A)$ $(M))$, the ground truths of corresponding original images $((B)-(N))$, outputs by Fu [ 37 ] ((C) (O)), Orlando [ 38 ] ((D) - (P)), Niemeijer [ 39 ] ((E) - (Q)) and our method ((F) - (R)), respectively. The places where are highlighted in original images respectively show the focused areas.

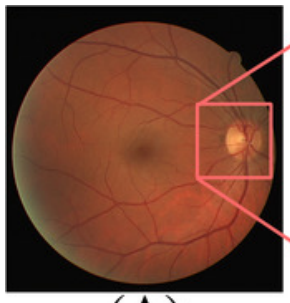

(A)

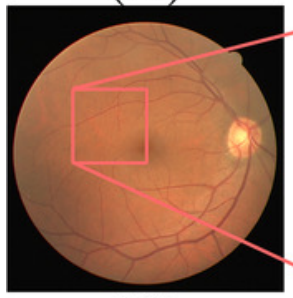

(G)

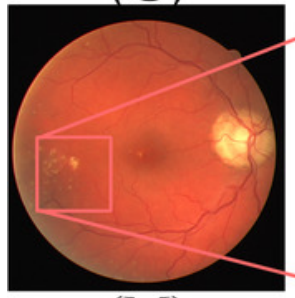

(M)

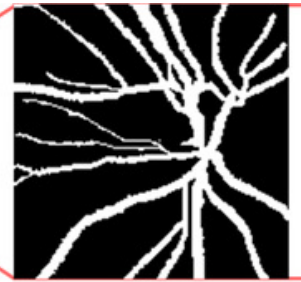

(B)

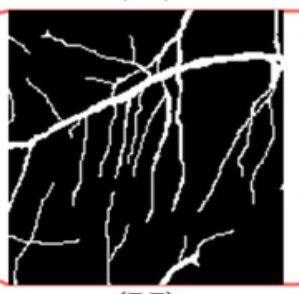

(H)

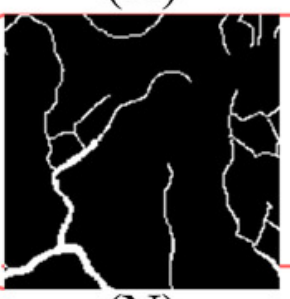

(N)

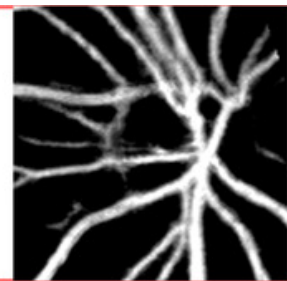

(C)

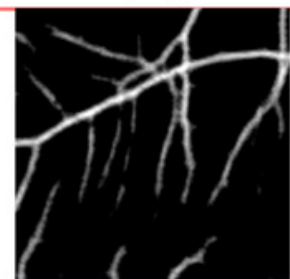

(I)

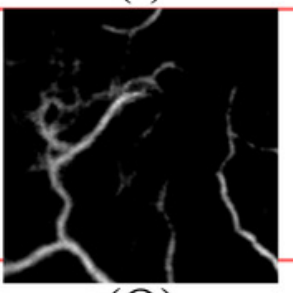

(O)

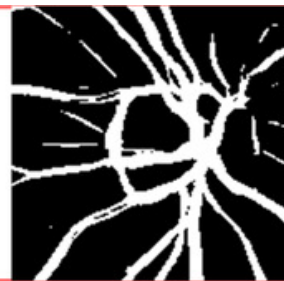

(D)

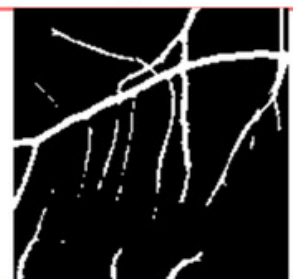

(J)

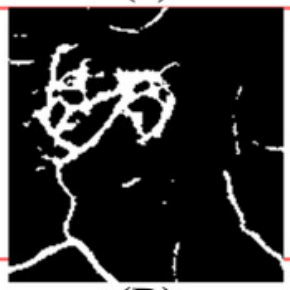

(P)

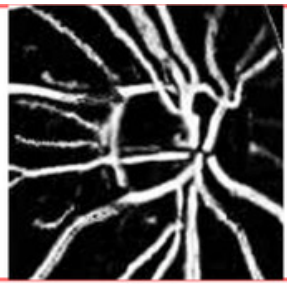

(E)

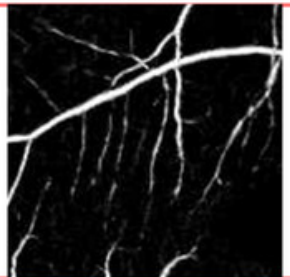

(K)

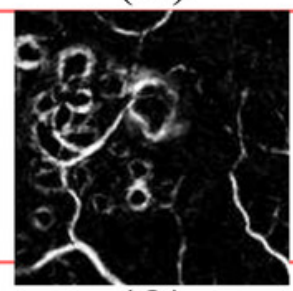

(Q)

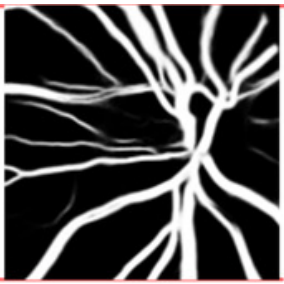

(F)

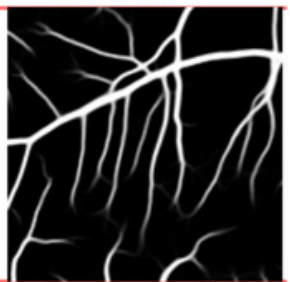

(L)

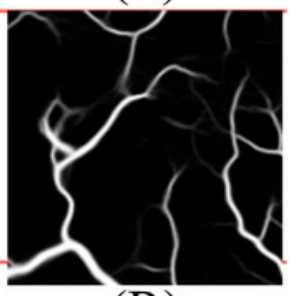

(R) 